\title{
Fundamental Problems of the Electrodynamics of Heterogeneous Media
}

\author{
N. N. Grinchik and Yu. N. Grinchik \\ A.V. Luikov Heat and Mass Transfer Institute of the National Academy of Sciences of Belarus, Minsk, Belarus 15, \\ P. Brovka Str., 2200072, Belarus
}

Correspondence should be addressed to N. N. Grinchik, nngrin@yandex.ru

Received 28 July 2011; Accepted 26 October 2011

Academic Editor: Vladimir Shavrov

Copyright ( $\odot 2012$ N. N. Grinchik and Yu. N. Grinchik. This is an open access article distributed under the Creative Commons Attribution License, which permits unrestricted use, distribution, and reproduction in any medium, provided the original work is properly cited.

The consistent physic-mathematical model of propagation of an electromagnetic wave in a heterogeneous medium is constructed using the generalized wave equation and the Dirichlet theorem. Twelve conditions at the interfaces of adjacent media are obtained and justified without using a surface charge and surface current in explicit form. The conditions are fulfilled automatically in each section of counting schemes for calculations. A consistent physicomathematical model of interaction of nonstationaly electric and thermal fields in a layered medium with allowance or mass transfer is constructed. The model is based on the methods of thermodynamics and on the equations of an electromagnetic field and is formulated without explicit separation of the charge carriers and the charge of an electric double layer.

\section{Introduction}

Let us consider the interface $S$ between two media having different electrophysical properties. On each of its sides the magnetic-field and magnetic-inductance vectors as well as the electric-field and electric-displacement vectors are finite and continuous; however, at the surface $S$ they can experience a discontinuity of the first kind. Furthermore, at the interface there arise induced surface charges $\sigma$ and surface currents $i$ (whose vectors lie in the plane tangential to the surface $S$ ) under the action of an external electric field.

The existence of a surface charge at the interface $S$ between the two media having different electrophysical properties is clearly demonstrated by the following example. We will consider the traverse of a direct current through a flat capacitor filled with two dielectric materials having relative permittivities $\varepsilon_{1}$ and $\varepsilon_{2}$ and electrical conductivities $\lambda_{1}$ and $\lambda_{2}$. A direct-current voltage $U$ is applied to the capacitor plates; the total resistance of the capacitor is $R$ (Figure 1). It is necessary to calculate the surface electric charge induced by the electric current.

From the electric-charge conservation law follows the constancy of flow in a circuit; therefore, the following equation is fulfilled:

$$
\lambda_{1} E_{n_{1}}=\lambda_{2} E_{n_{2}}=\frac{U}{(R S)},
$$

where $E_{n_{1}}$ and $E_{n_{2}}$ are the normal components of the electricfield vector.

At the interface between the dielectrics, the normal components of the electric-inductance vector change spasmodically under the action of the electric field by a value equal to the value of the induced surface charge $\sigma$ :

$$
\mathcal{E}_{0} \varepsilon_{1} E_{n_{1}}-\varepsilon_{0} \varepsilon_{2} E_{n_{2}}=\sigma .
$$


Solving the system of (1) and (2), we obtain the expression for $\sigma$ :

$$
\sigma=\left(\frac{U}{R S}\right) \varepsilon_{0}\left[\left(\frac{\varepsilon_{1}}{\lambda_{1}}\right)-\left(\frac{\varepsilon_{2}}{\lambda_{2}}\right)\right] .
$$

It follows from (3) that the charge $\sigma$ is determined by the current and the multiplier accounting for the properties of the medium. If

$$
\left(\frac{\varepsilon_{1}}{\lambda_{1}}\right)-\left(\frac{\varepsilon_{2}}{\lambda_{2}}\right)=0,
$$

a surface charge $\sigma$ is not formed. What is more, recent trends are toward increased use of micromachines and engines made from plastic materials, where the appearance of a surface charge is undesirable. For oiling of elements of such machines, it is best to use an oil with a permittivity $\varepsilon_{\text {oil }}$ satisfying the relation

$$
\varepsilon_{1}<\varepsilon_{\text {oil }}<\varepsilon_{2}
$$

This oil makes it possible to decrease the electrization of the moving machine parts made from dielectric materials. In addition to the charge $\sigma$, a contact potential difference arises always independently of the current.

An electric field interacting with a material is investigated with the use of the Maxwell equation (1857). For convenience and correctness of further presentation we denote the axial vector of magnetic field strength, induction as $\vec{H}$ and $\vec{B}$

$$
\begin{array}{ll}
\mathbf{j}_{\text {total }}=\nabla \times \overrightarrow{\mathbf{H}}, & \nabla \cdot \mathbf{D}=\rho, \\
-\frac{\partial \overrightarrow{\mathbf{B}}}{\partial t}=\nabla \times \mathbf{E}, & \nabla \cdot \overrightarrow{\mathbf{B}}=0,
\end{array}
$$

where $\mathbf{j}_{\text {total }}=\lambda \mathbf{E}+\partial \overrightarrow{\mathbf{D}} / \partial t, \overrightarrow{\mathbf{B}}=\mu \mu_{0} \overrightarrow{\mathbf{H}}$. In this case, at the interface $S$ the above system of equations is supplemented with the boundary conditions $[1,2]$ :

$$
\begin{aligned}
D_{n_{1}}-D_{n_{2}} & =\sigma, \\
E_{\tau_{1}}-E_{\tau_{2}} & =0, \\
B_{n_{1}}-B_{n_{2}} & =0, \\
\overrightarrow{\mathbf{H}}_{\tau_{1}}-\overrightarrow{\mathbf{H}}_{\tau_{2}} & =[\vec{\imath} \cdot \vec{n}] .
\end{aligned}
$$

The indices (subscripts) $n$ and $\tau$ denote the normal and tangential components of the vectors to the surface $S$, and the indices 1 and 2 denote the adjacent media with different electrophysical properties. The index $\tau$ denotes any direction tangential to the discontinuity surface. At the same time, a closing relation is absent for the induced surface charge $\sigma$, which generates a need for the introduction of an impedance matrix [3-7] that is determined experimentally or, in some cases, theoretically from quantum representations [8-12]. We note that the Maxwell equation of electromagnetic field does not coincide completely with the modern form of them (6), (7) which was first given by Hertz (1884) and independently of him by Heaviside (1885). The equations

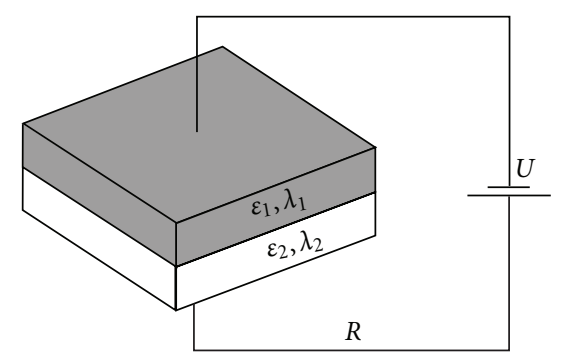

FIGURE 1: Dielectric media inside a flat capacitor.

of electromagnetic field with precise division of polar and axial vectors are of importance in considering the boundary conditions. One should bear in mind that electric field strength $\mathbf{E}$ is a polar vector. Gradients of concentration and temperature are also polar vectors. On the contrary, magnetic field strength $\overrightarrow{\mathbf{H}}$, as well as $\overrightarrow{\mathbf{H}}$, are axial vectors, or pseudovectors. This is well seen from the formula for the Lorentz force $\mathbf{F}=q[\mathbf{V} \overrightarrow{\mathbf{H}}]$. Indeed, the behavior of $\overrightarrow{\mathbf{H}}$ on reflection at the origin of coordinates and substitution $\mathbf{r} \rightarrow$ $(-\mathbf{r})$ is determined by the behavior of the polar vectors $\mathbf{F}$ and $\mathrm{V}$ and the properties of their vector product. On substitution $\mathbf{r} \rightarrow(-\mathbf{r})$ the directions of the vectors $\mathbf{F}$ and $\mathbf{V}$ change to the opposite; the sign of the vector product also reverses. Consequently, on substitution $\mathbf{r} \rightarrow(-\mathbf{r})$ the vector $\overrightarrow{\mathbf{H}}$ must remain constant. This property is assumed to be an attribute of the axial vector.

The induced surface charge $\sigma$ not only characterizes the properties of a surface, but also represents a function of the process, that is, $\sigma(\mathbf{E}(\partial \mathbf{E} / \partial t, \overrightarrow{\mathbf{H}}(\partial \overrightarrow{\mathbf{H}} / \partial t)))$; therefore, the surface impedances [3-7] are true for the conditions under which they are determined. These impedances cannot be used in experiments conducted under other experimental conditions.

The problem of determination of surface charge and surface current on metal-electrolyte boundaries becomes even more complicated in investigating and modeling nonstationary electrochemical processes, for example, pulse electrolysis, when lumped parameters $L, C$, and $R$ cannot be used in principle.

We will show that $\sigma$ can be calculated using the Maxwell phenomenological macroscopic electromagnetic equations and the electric-charge conservation law accounting for the special features of the interface between the adjacent media.

Separate consideration will be given to ion conductors. In constructing a physicomathematical model, we take into account that $\mathbf{E}$ and $\overrightarrow{\mathbf{H}}$ are not independent functions; therefore, the wave equation for $\mathbf{E}$ or $\overrightarrow{\mathbf{H}}$ is more preferable than the system of (6) and (7). It will be shown that in consideration of propagation, interference of waves in the heterogeneous medium it is preferable to use the wave equation for a polar vector of electric field strength. 


\section{Electron Conductors: New Closing Relations on the Boundaries of Adjacent Media}

2.1. Generalized Wave Equation for $E$ and Conditions on the Boundaries in the Presence of Strong Discontinuities of the Electromagnetic Field

2.1.1. Physicomathematical Model. We will formulate a physicomathematical model of propagation of an electromagnetic field in a heterogeneous medium. Let us multiply the left and right sides of the equation for the total current (see (6)) by $\mu \mu_{0}$ and differentiate it with respect to time. Acting by the operator rot on the left and right sides of the first equation of ( 7 ) on condition that $\mu=$ const we obtain

$$
\frac{\partial \mathbf{j}_{\text {total }}}{\partial t}=\frac{1}{\mu \mu_{0}} \nabla^{2} \mathbf{E}-\frac{1}{\mu \mu_{0}} \operatorname{grad}(\operatorname{div} \mathbf{E}) .
$$

In Cartesian coordinates, (12) will take the form

$$
\begin{aligned}
& \frac{\partial \mathbf{j}_{\text {total } x}}{\partial t}= \frac{1}{\mu \mu_{0}}\left(\frac{\partial^{2} E_{x}}{\partial x^{2}}+\frac{\partial^{2} E_{x}}{\partial y^{2}}+\frac{\partial^{2} E_{x}}{\partial z^{2}}\right) \\
&-\frac{1}{\mu \mu_{0}} \frac{\partial}{\partial x}\left(\frac{\partial E_{x}}{\partial x}+\frac{\partial E_{y}}{\partial y}+\frac{\partial E_{z}}{\partial z}\right), \\
& \frac{\partial \mathbf{j}_{\text {total } y}}{\partial t}= \frac{1}{\mu \mu_{0}}\left(\frac{\partial^{2} E_{y}}{\partial x^{2}}+\frac{\partial^{2} E_{y}}{\partial y^{2}}+\frac{\partial^{2} E_{y}}{\partial z^{2}}\right) \\
&-\frac{1}{\mu \mu_{0}} \frac{\partial}{\partial y}\left(\frac{\partial E_{x}}{\partial x}+\frac{\partial E_{y}}{\partial y}+\frac{\partial E_{z}}{\partial z}\right), \\
& \frac{\partial \mathbf{j}_{\text {total } z}=}{\partial t}=\frac{1}{\mu \mu_{0}}\left(\frac{\partial^{2} E_{z}}{\partial x^{2}}+\frac{\partial^{2} E_{z}}{\partial y^{2}}+\frac{\partial^{2} E_{z}}{\partial z^{2}}\right) \\
&-\frac{1}{\mu \mu_{0}} \frac{\partial}{\partial z}\left(\frac{\partial E_{x}}{\partial x}+\frac{\partial E_{y}}{\partial y}+\frac{\partial E_{z}}{\partial z}\right) .
\end{aligned}
$$

At the interface, the following relation [2] is also true:

$$
\operatorname{div} \mathbf{i}_{\tau}+I_{q x_{1}}-I_{q x_{2}}=-\frac{\partial \sigma}{\partial t}
$$

Let us write conditions (see (8)-(11)) in the Cartesian coordinate system:

$$
\begin{gathered}
D_{x_{1}}-D_{x_{2}}=\sigma, \\
E_{y_{1}}-E_{y_{2}}=0, \\
E_{z_{1}}-E_{z_{2}}=0, \\
B_{x_{1}}-B_{x_{2}}=0, \\
H_{y_{1}}-H_{y_{2}}=i_{z}, \\
H_{z_{1}}-H_{z_{2}}=i_{y},
\end{gathered}
$$

where $\mathbf{i}_{\tau}=i_{y} \mathbf{j}+i_{z} \mathbf{k}$ is the surface-current density, and the coordinate $x$ is directed along the normal to the interface. The densities $i_{y}$ and $i_{z}$ of the surface currents represent the electric charge carried in unit time by a segment of unit length positioned on the surface drawing the current perpendicularly to its direction.
The order of the system of differential equations (13) is equal to (16). Therefore, at the interface $S$, it is necessary to set, by and large, nine boundary conditions. Moreover, the three additional conditions (15), (19), and (20) containing (prior to the solution) unknown quantities should be fulfilled at this interface. Consequently, the total number of conjugation conditions at the boundary $S$ should be equal to (12) for a correct solution of the problem.

As an example we consider a one-dimensional case of heat propagation in the layered material. As is known, to solve the problem we must specify two conditions at the interface: the equality of heat fluxes and the equality of temperatures, since in the heat conduction equation we have the second derivative with respect to the coordinate.

Differentiating expression (15) with respect to time and using relation (14), we obtain the following condition for the normal components of the total current at the mediummedium interface:

$$
\operatorname{div} \mathbf{i}_{\tau}+\mathbf{j}_{\text {total } x_{1}}=\mathbf{j}_{\text {total } x_{2}},
$$

that allows one to disregard the surface charge $\sigma$. Let us introduce the arbitrary function $f:\left.[f]\right|_{x=\xi}=\left.f_{1}\right|_{x=\xi+0}-$ $\left.f_{2}\right|_{x=\xi-0}$. In this case, expression (21) will take the form

$$
\left.\left[\operatorname{div} \mathbf{i}_{\tau}+\mathbf{j}_{\text {total } x_{1}}\right]\right|_{x=\xi}=0
$$

It is assumed that, at the medium-medium interface, $E_{x}$ is a continuous function of $y$ and $z$. Then, differentiating Equation (21) with respect to $y$ and $z$, we obtain

$$
\begin{aligned}
& {\left.\left[\frac{\partial}{\partial y} \mathbf{j}_{\text {total } x_{1}}\right]\right|_{x=\xi}=-\frac{\partial\left(\operatorname{div} \mathbf{i}_{\tau}\right)}{\partial y},} \\
& {\left.\left[\frac{\partial}{\partial z} \mathbf{j}_{\text {total } x_{1}}\right]\right|_{x=\xi}=-\frac{\partial\left(\operatorname{div} \mathbf{i}_{\tau}\right)}{\partial z} .}
\end{aligned}
$$

Let us differentiate conditions (18)-(20) for the magnetic induction and the magnetic-field strength with respect to time. On condition that $\overrightarrow{\mathbf{B}}=\mu \mu_{0} \overrightarrow{\mathbf{H}}$ :

$$
\begin{gathered}
{\left.\left[\frac{\partial B_{x}}{\partial t}\right]\right|_{x=\xi}=0,} \\
{\left.\left[\frac{1}{\mu \mu_{0}} \frac{\partial B_{y}}{\partial t}\right]\right|_{x=\xi}=\frac{\partial i_{z}}{\partial t},} \\
{\left.\left[\frac{1}{\mu \mu_{0}} \frac{\partial B_{z}}{\partial t}\right]\right|_{x=\xi}=\frac{\partial i_{y}}{\partial t} .}
\end{gathered}
$$


Using (7) and expressing (24) in terms of projections of the electric-field rotor, we obtain

$$
\begin{gathered}
{\left.\left[\operatorname{rot}_{x} \mathbf{E}\right]\right|_{x=\xi}=0,\left.\quad\left[\frac{\partial E_{z}}{\partial y}-\frac{\partial E_{y}}{\partial z}\right]\right|_{x=\xi}=0,} \\
{\left.\left[\frac{1}{\mu \mu_{0}} \operatorname{rot}_{y} \mathbf{E}\right]\right|_{x=\xi}=\frac{\partial i_{z}}{\partial t}} \\
\text { or }\left.\left[\frac{1}{\mu \mu_{0}}\left(\frac{\partial E_{x}}{\partial z}-\frac{\partial E_{z}}{\partial x}\right)\right]\right|_{x=\xi}=\frac{\partial i_{z}}{\partial t} \\
{\left.\left[\frac{1}{\mu \mu_{0}} \operatorname{rot}_{z} \mathbf{E}\right]\right|_{x=\xi}=\frac{\partial i_{z}}{\partial t}} \\
\text { or }\left.\left[\frac{1}{\mu \mu_{0}}\left(\frac{\partial E_{y}}{\partial x}-\frac{\partial E_{x}}{\partial y}\right)\right]\right|_{x=\xi}=\frac{\partial i_{z}}{\partial t}
\end{gathered}
$$

Here, (25) is the normal projection of the electric-field rotor, (26) is the tangential projection of the rotor on $y$, and (27) is the rotor projection on $z$.

Assuming that $E_{y}$ and $E_{z}$ are continuous differentiable functions of the coordinates $y$ and $z$, from conditions (16) and (17) we find

$$
\begin{array}{ll}
{\left.\left[\frac{\partial E_{y}}{\partial y}\right]\right|_{x=\xi}=0,} & {\left.\left[\frac{\partial E_{y}}{\partial z}\right]\right|_{x=\xi}=0,} \\
{\left.\left[\frac{\partial E_{z}}{\partial y}\right]\right|_{x=\xi}=0,} & {\left.\left[\frac{\partial E_{z}}{\partial z}\right]\right|_{x=\xi}=0 .}
\end{array}
$$

In accordance with the condition that the tangential projections of the electric field on $z$ and $y$ are equal and in accordance with conditions (16) and (17), the expressions for the densities of the surface currents $i_{z}$ and $i_{y}$ take the form

$$
i_{z}=\left.\bar{\lambda} E_{z}\right|_{x=\xi}, \quad i_{y}=\left.\bar{\lambda} E_{y}\right|_{x=\xi},
$$

where

$$
\bar{\lambda}=\left.\frac{1}{2}\left(\lambda_{1}+\lambda_{2}\right)\right|_{x=\xi}
$$

is the average value of the electrical conductivity at the interface between the adjacent media in accordance with the Dirichlet theorem for a piecewise-smooth, piecewisedifferentiable function.

Consequently, formulas (28)-(30) yield

$$
\left.\left[\operatorname{div} \overrightarrow{i_{\tau}}\right]\right|_{x=\xi}=0 .
$$

Relations (31) and (32) and hence the equality of the normal components of the total current were obtained (in a different manner) by Grinberg and Fok [13]. In this work, it has been shown that condition (31) leads to the equality of the derivatives of the electric field strength along the normal to the surface

$$
\left.\left[\frac{\partial E_{x}}{\partial x}\right]\right|_{x=\xi}=0 .
$$

With allowance for the foregoing we have twelve conditions at the interface between the adjacent media that are necessary for solving the complete system of (13): (a) the functions $E_{y}$ and $E_{z}$ are determined from (16) and (17);

(b) $E_{x}$ is determined from condition (22);

(c) the values of $\partial E_{x} / \partial y, \partial E_{x} / \partial z$, and $\partial E_{x} / \partial x$ are determined from relations (23) with the use of the condition of continuity of the total-current normal component at the interface (22) and the continuity of the derivative of the total current with respect to the coordinate $x$;

(d) the values of $\partial E_{y} / \partial y, \partial E_{y} / \partial z$, and $\partial E_{z} / \partial z$ are determined from conditions (28) and (29) in consequence of the continuity of the tangential components of the electric field along $y$ and $z$;

(e) the derivatives $\partial E_{y} / \partial x$ and $\partial E_{z} / \partial x$ are determined from conditions (26) and (27) as a consequence of the equality of the tangential components of the electricfield rotor along $y$ and $z$.

Note that condition (21) was used by us in [14] in the numerical simulation of the pulsed electrochemical processes in the one-dimensional case. Condition (25) for the normal component of the electric-field rotor represents a linear combination of conditions (28) and (29); therefore, $\operatorname{rot}_{x} E=0$ and there is no need to use it in the subsequent discussion. The specificity of the expression for the general law of electric-charge conservation at the interface is that the components $\partial E_{y} / \partial y$ and $\partial E_{z} / \partial z$ are determined from conditions (28) and (29) that follow from the equality and continuity of the tangential components $E_{y}$ and $E_{z}$ at the boundary $S$.

Thus, at the interface between the adjacent media the following conditions are fulfilled: the equality of the totalcurrent normal components; the equality of the tangential projections of the electric-field rotor; the electric-charge conservation law; the equality of the electric-field tangential components and their derivatives in the tangential direction; the equality of the derivatives of the total-current normal components in the direction tangential to the interface between the adjacent media, determined with account for the surface currents and without explicit introduction of a surface charge. They are true at each cross section of the sample being investigated.

2.1.2. Features of Calculation of the Propagation of Electromagnetic Waves in Layered Media. The electromagnetic effects arising at the interface between different media under the action of plane electromagnetic waves have a profound impact on the equipment because all real devices are bounded by the surfaces and are inhomogeneous in space. At the same time, the study of the propagation of waves in layered conducting media and, according to [15], in thin films is reduced to the calculation of the reflection and transmission coefficients; the function $E(x)$ is not determined in the thickness of a film, that is, the geometrical-optics approximation is used.

The physicomathematical model proposed allows one to investigate the propagation of an electromagnetic wave in a 
layered medium without recourse to the assumptions used in $[3-5,7]$.

Since conditions (21)-(29) are true at each cross section of a layered medium, we will use schemes of through counting without an explicit definition of the interface between the media. In this case, it is proposed to calculate $E_{x}$ at the interface in the following way.

In accordance with (15), $E_{x 1} \neq E_{x 2}$, that is, $E_{x}(x)$ experiences a discontinuity of the first kind. Let us determine the strength of the electric field at the discontinuity point $x=\xi$ on condition that $E_{x}(x)$ is a piecewise-smooth, piecewisedifferentiable function having finite one-sided derivatives $E_{x^{+}}^{\prime}(x)$ and $E_{x^{-}}^{\prime}(x)$. At the discontinuity points $x_{i}$,

$$
\begin{aligned}
& E_{x^{+}}^{\prime}\left(x_{i}\right)=\lim _{\Delta x_{i} \rightarrow+0} \frac{E\left(x_{i}+\Delta x_{i}\right)-E\left(x_{i}+0\right)}{\Delta x_{i}}, \\
& E_{x^{-}}^{\prime}\left(x_{i}\right)=\lim _{\Delta x_{i} \rightarrow-0} \frac{E\left(x_{i}+\Delta x_{i}\right)-E\left(x_{i}-0\right)}{\Delta x_{i}} .
\end{aligned}
$$

In this case, in accordance with the Dirichlet theorem [16], the Fourier series of the function $E(x)$ at each point $x$, including the discontinuity point $\xi$, converges and its sum is equal to

$$
E_{x=\xi}=\frac{1}{2}[E(\xi-0)+E(\xi+0)] .
$$

The Dirichlet condition (34) also has a physical meaning. In the case of contact of two solid conductors, for example, dielectrics or electrolytes in different combinations (metalelectrolyte, dielectric-electrolyte, metal-vacuum, and so on), at the interface between the adjacent media there always arises an electric double-layer (EDL) with an unknown (as a rule) structure that, however, substantially influences the electrokinetic effects, the rate of the electrochemical processes, and so on. It is significant that, in reality, the electrophysical characteristics $\lambda, \varepsilon$, and $E(x)$ change uninterruptedly in the electric double-layer; therefore, (34) is true for the case where the thickness of the electric doublelayer, that is, the thickness of the interphase boundary, is much smaller than the characteristic size of a homogeneous medium. In a composite, for example, in a metal with embedments of dielectric balls, where the concentration of both components is fairly large and their characteristic sizes are small, the interphase boundaries can overlap and condition (34) can break down.

If the thickness of the electric double-layer is much smaller than the characteristic size $L$ of an object, (34) also follows from the condition that $E(x)$ changes linearly in the EDL region. In reality, the thickness of the electric doublelayer depends on the kind of contacting materials and can comprise several tens of angstroms [17]. In accordance with the modern views, the outer coat of the electric double-layer consists of two parts, the first of which is formed by the ions immediately attracted to the surface of the metal (a "dense" or a "Helmholtz" layer of thickness $h$ ), and the second is formed by the ions separated by distances larger than the ion radius from the surface of the layer, and the number of these ions decreases as the distance between them and the interface (the "diffusion layer") increases. The distribution of the potential in the dense and diffusion parts of the electric double-layer is exponential in actual practice [17], that is, the condition that $E(x)$ changes linearly breaks down; in this case, the sum of the charges of the dense and diffusion parts of the outer coat of the electric double-layer is equal to the charge of its inner coat (the metal surface). However, if the thickness of the electric double-layer $h$ is much smaller than the characteristic size of an object, the expansion of $E(x)$ into a power series is valid and one can restrict oneself to the consideration of a linear approximation. In accordance with the more general Dirichlet theorem (1829), a knowledge of this function in the EDL region is not necessary to substantiate (34). Nonetheless, the above-indicated physical features of the electric double-layer lend support to the validity of condition (34).

The condition at interfaces, analogous to (34), has been obtained earlier [18] for the potential field (where $\operatorname{rot} \mathbf{E}=$ 0 ) on the basis of introduction of the surface potential, the use of the Green formula, and the consideration of the discontinuity of the potential of the double-layer. In [18], it is also noted that the consideration of the thickness of the double-layer and the change in its potential at $h / L \ll$ 1 makes no sense in general; therefore, it is advantageous to consider, instead of the volume potential, the surface potential of any density. Condition (34) can be obtained, as was shown in [16], from the more general Dirichlet theorem for a nonpotential vorticity field [18].

Thus, the foregoing and the validity of conditions (15)(17) and (23)-(29) at each cross-section of a layered medium show that, for numerical solution of the problem being considered it is advantageous to use schemes of through counting and make the discretization of the medium in such a way that the boundaries of the layers have common points.

The medium was divided into finite elements so that the nodes of a finite-element grid, lying on the separation surface between the media with different electrophysical properties, were shared by these media at a time. In this case, the total currents or the current flows at the interface should be equal if the Dirichlet condition (34) is fulfilled.

\subsubsection{Results of Numerical Simulation of the Propagation of} Electromagnetic Waves in Layered Media. Let us analyze the propagation of an electromagnetic wave through a layered medium that consists of several layers with different electrophysical properties in the case where an electromagneticradiation source is positioned on the upper plane of the medium. It is assumed that the normal component of the electric-field vector $E_{x}=0$ and its tangential component $E_{y}=a \sin (\omega t)$, where $a$ is the electromagnetic-wave amplitude (Figure 2).

In this example, for the purpose of correct specification of the conditions at the lower boundary of the medium, an additional layer is introduced downstream of layer 6; this layer has a larger conductivity and, therefore, the electromagnetic wave is damped out rapidly in it. In this case, the condition $E_{y}=E_{z}=0$ can be set at the lower boundary of the medium. The above manipulations were made to limit the size of the medium being considered because, 


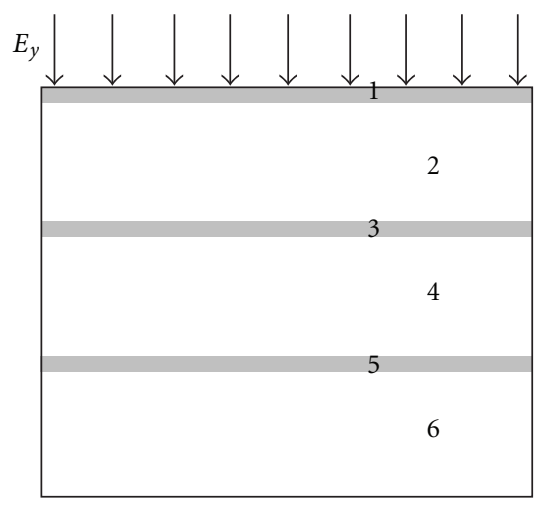

Figure 2: Scheme of a layered medium: layers 1, 3, and 5 are characterized by the electrophysical parameters $\varepsilon_{1}, \lambda_{1}$, and $\mu_{1}$, and layers 2,4 , and 6 by $\varepsilon_{2}, \lambda_{2}$, and $\mu_{2}$.

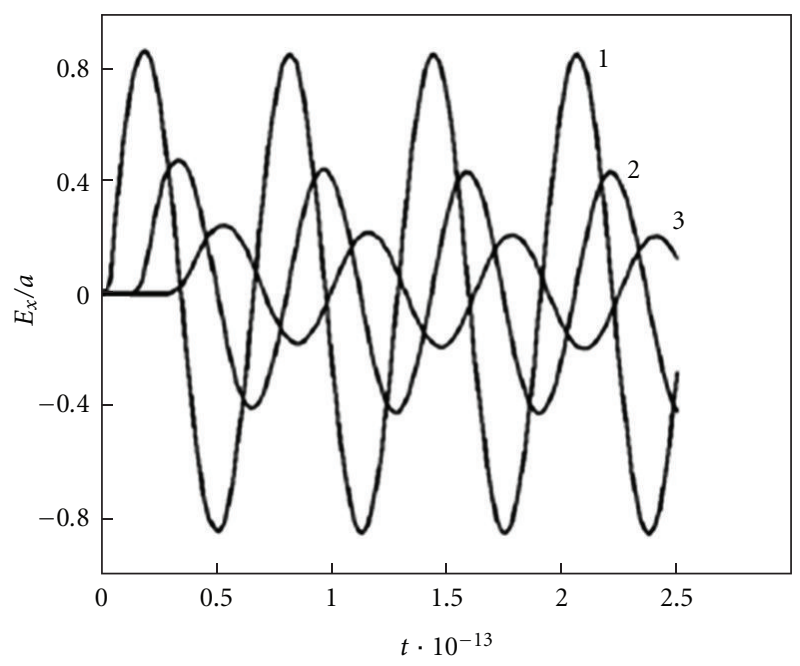

FIGURE 3: Time change in the tangential component of the electricfield strength at a distance of $1 \mu \mathrm{m}$ (1), $5 \mu \mathrm{m}$ (2), and $10 \mu \mathrm{m}$ (3) from the surface of the medium at $\lambda_{1}=100, \lambda_{2}=1000, \varepsilon_{1}=\varepsilon_{2}=1$, $\mu_{1}=\mu_{2}=1$, and $\omega=10^{14} \mathrm{~Hz}$. $t$, sec.

in the general case, the electromagnetic wave is attenuated completely at an infinite distance from the electromagneticradiation source.

Numerical calculations of the propagation of an electromagnetic wave in the layered medium with electrophysical parameters $\varepsilon_{1}=\varepsilon_{2}=1, \lambda_{1}=100, \lambda_{2}=$ 1000 , and $\mu_{1}=\mu_{2}=1$ were carried out. Two values of the cyclic frequency $\omega=2 \pi / T$ were used: in the first case, the electromagnetic-wave frequency was assumed to be equal to $\omega=10^{14} \mathrm{~Hz}$ (infrared radiation), and, in the second case, the cyclic frequency was taken to be $\omega=10^{9} \mathrm{~Hz}$ (radiofrequency radiation).

As a result of the numerical solution of the system of (13) with the use of conditions $S$ (22)-((31) and (32) at the interfaces, we obtained the time dependences of the electricfield strength at different distances from the surface of the layered medium (Figure 3).

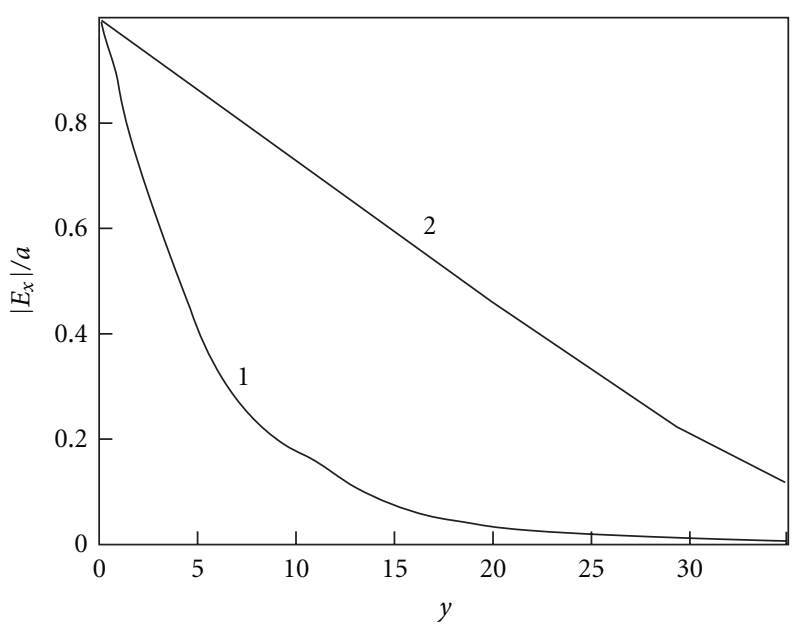

Figure 4: Distribution of the amplitude of the electric-fieldstrength at the cross section of the layered medium: $\omega=10^{14}(1)$ and $10^{9} \mathrm{~Hz}(2) . y, \mu \mathrm{m}$.

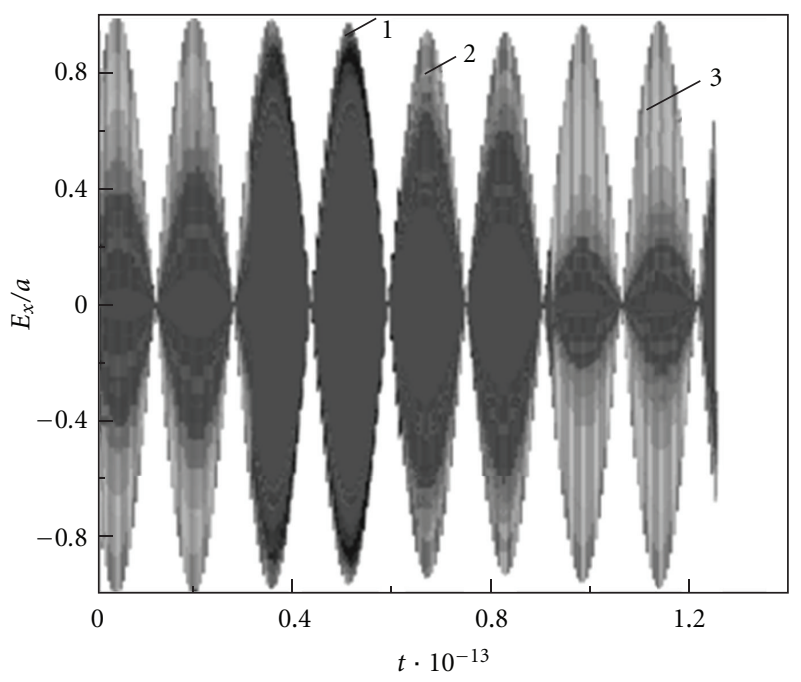

FIGURE 5: Time change in the electric-field strength at a distance of 1 (1), 5 (2), and $10 \mu \mathrm{m}$ (3) from the surface of the medium. $t$, sec.

The results of our simulation (Figure 4) have shown that a high-frequency electromagnetic wave propagating in a layered medium is damped out rapidly, whereas a low-frequency electromagnetic wave penetrates into such a medium to a greater depth. The model developed was also used for calculating the propagation of a modulated signal of frequency $20 \mathrm{kHz}$ in a layered medium. As a result of our simulation (Figure 5), we obtained changes in the electric-field strength at different depths of the layered medium, which points to the fact that the model proposed can be used to advantage for calculating the propagation of polyharmonic waves in layered media; such a calculation cannot be performed on the basis of the Helmholtz equation.

The physicomathematical model developed can also be used to advantage for simulation of the propagation of electromagnetic waves in media with complex geometric 
parameters and large discontinuities of the electromagnetic field (Figure 6).

Figure 6(a) shows the cross-sectional view of a cellular structure representing a set of parallelepipeds with different cross-sections in the form of squares. The parameters of the materials in the large parallelepiped are denoted by Index 1, and the parameters of the materials in the small parallelepipeds (the squares in the figure) are denoted by Index 2.

An electromagnetic wave propagates in the parallelepipeds (channels) in the transverse direction. It is seen from Figure 6(b) that, in the cellular structure there are "silence regions," where the amplitude of the electromagnetic-wave strength is close to zero, as well as inner regions where the signal has a marked value downstream of the "silence" zone formed as a result of the interference.

2.1.4. Results of Numerical Simulation of the Scattering of Electromagnetic Waves in Angular Structures. It is radiolocation and radio-communication problems that are among the main challenges in the set of problems solved using radioengineering devices.

Knowledge of the space-time characteristics of diffraction fields of electromagnetic waves scattered by an object of location into the environment is necessary for solving successfully any radiolocation problem. Irradiated objects have a very intricate architecture and geometric shape of the surface consisting of smooth portions and numerous wedgeshaped for formations of different type-angular joints of smooth portions, surface fractures, sharp edges, and so forth, with rounded radii much smaller than the probing-signal wavelength. Therefore, solution of radiolocation problems requires the methods of calculation of the diffraction fields of electromagnetic waves excited and scattered by different surface portions of the objects, in particular, by wedgeshaped formations, be known, since the latter are among the main sources of scattered waves.

For another topical problem, that is, radio communication effected between objects, the most difficult are the issues of designing of antennas arranged on an object, since their operating efficiency is closely related to the geometric and radiophysical properties of its surface.

The issues of diffraction of an electromagnetic wave in wedge-shaped regions are the focus of numerous of the problems for a perfectly conducting and impedance wedge for monochromatic waves is representation of the diffraction field in an angular region in the form of a Sommerfeld integral [19].

Substitution of Sommerfeld integrals into the system of boundary conditions gives a system of recurrence functional equations for unknown analytical integrands. The system's coefficients are Fresnel coefficients defining the reflection of plane media or their refraction into the opposite medium. From the system of functional equations, one determines, in a recurrence manner, sequences of integrand poles and residues in these poles.
The edge diffraction field in both media is determined using a pair of Fredholm-type singular integral equations of the second kind which are obtained from the aboveindicated systems of functional equations with subsequent computation of Sommerfeld integrals by the saddle-point approximation. The branching points of the integrands condition the presence of creeping waves excited by the edge of the dielectric wedge.

The proposed method is only true of monochromatic waves and of the approximate Leontovich boundary conditions, when the field of the electromagnetic wave slowly varies from point to point on a wavelength scale [20].

We note that the existing approximate Leontovich conditions have a number of other constants and should be used with caution [20].

In actual fact, the proposed calculation method does not work in the presence of, for example, two wedges, when the sharp angles are pointed at each other, that is, an optical knife, or in diffraction of the electromagnetic wave on a system of parallel lobes, when the gap between the lobes is in the region of microns, and the electromagnetic field is strongly "cut" throughout the space with a step much than the wavelength.

(A) Optical Knife. Figure 7 shows the field of an electromagnetic wave in its diffraction on the optical knife. The parameters of the wave at entry and at exit are $E_{x}=$ $10^{4} \sin \left(10^{10} t\right), E_{y}=10^{4} \cos \left(10^{10} t\right)$.

The electrophysical characteristics are as follows. The wedge is manufactured from aluminum: $\varepsilon=1 ; \mu=1$; $\sigma=3.774 \cdot 10^{7} \mathrm{~S} / \mathrm{m}$; the ambient medium is air.

The dimensions of the computational domain are $0.1 \times$ $0.05 \mathrm{~m}$. The calculation time $10^{-9} \mathrm{sec}$, and the time step is $10^{-11} \mathrm{sec}$.

Numerical solution of the system of equations (13) yields the dependences of the distribution $E_{x}, E_{y}$ on the optical knife. The calculation results are in good agreement with the existing experimental data and experiments specially conducted at the Department of the Physics and Chemistry of Nonequilibrium Media of the A.V. Luikov Heat and Mass Transfer Institute of the National Academy of Sciences by A. I. Bereznyak.

The experiments were carried out with an optical-range laser and were tentative in character but the obtained experimental photographs of diffraction fields, and the calculated results turned out to be in good qualitative agreement. The authors express their thanks to A. I. Bereznyak for the conducting of the experiments.

(B) Diffraction Grating. The parameters of the wave and the interfacial conditions are the same, as those for the case "optical knife." The electrophysical characteristics are as follows: $2 \mathrm{D}$ lobes, $\varepsilon=12$; and $\sigma=100 \mathrm{~S} / \mathrm{m}$; the ambient medium is air; the characteristics of the prism and the square are identical to those of the lobes.

Figure 8 corresponds to a calculation time of $10^{-10} \mathrm{sec}$; the time step is $10^{-12} \mathrm{sec}$. Figure 9 corresponds to a calculation time of $10^{-9} \mathrm{sec}$; the time step is $10^{-11} \mathrm{sec}$. 


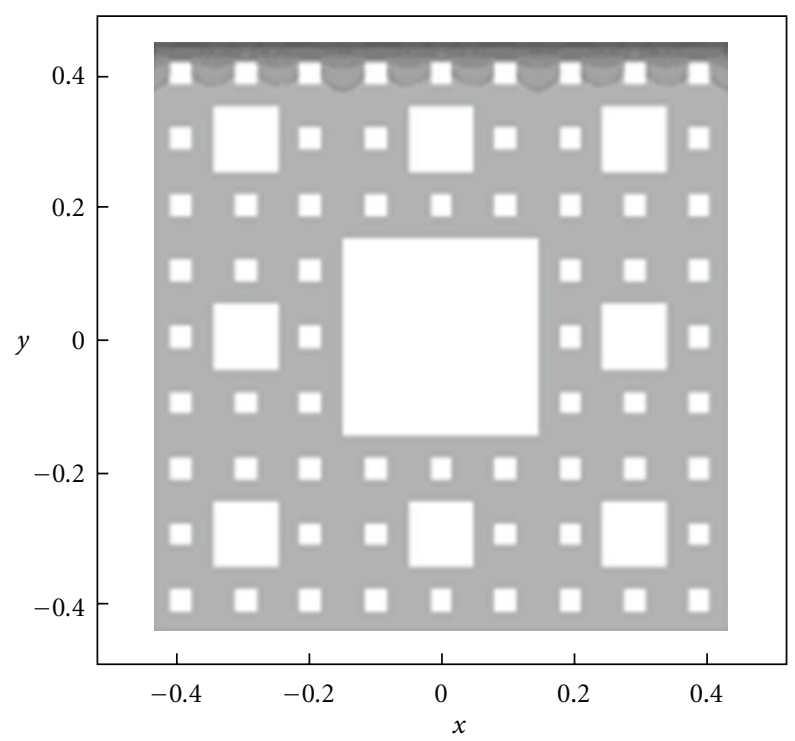

(a) Distribution of the amplitude of the electric-field strength in the two-dimensional medium

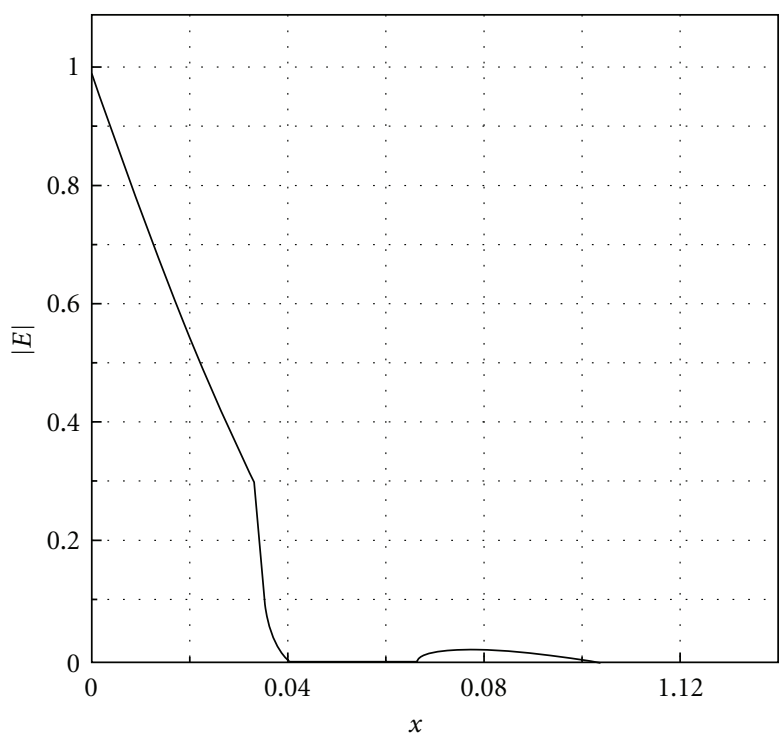

(b) Distribution of the amplitude of the electric-field strength in depth

Figure 6: Distribution of the amplitude of the electric-field strength in the two-dimensional medium and in depth at $\varepsilon_{1}=15$, $\varepsilon_{2}=20$, $\lambda_{1}=10^{-6}, \lambda_{2}=10, \mu_{1}=\mu_{2}=1$, and $\omega=10^{9} \mathrm{~Hz}$ (the dark background denotes medium 1, and the light background denotes medium 2). $x$, $y, \mathrm{~mm} ; E, \mathrm{~V} / \mathrm{m}$.

It is seen from the modeling results that the proposed "comb" cab be used as a filter of a high-frequency signal. Furthermore, we carried out numerical calculations of a modulated signal at a frequency of $20 \mathrm{kHz}$. The results of the modulated-signal calculations are not given. To analyze the difference scheme for stability was analyzed by the initial data. When the time and space steps are large there appear oscillations of the grid solution and of its "derivatives" ("ripple") which strongly decrease the accuracy of the scheme. Undoubtedly, this issue calls for separate consideration. The proposed algorithm of solution of Maxwell equations allows circuitry-engineering modeling of high-frequency radioengineering devices and investigation of the propagation of electromagnetic waves in media of intricate geometry in the presence of strong discontinuities of electromagnetic field.

The result of Para 2.1 were published in part [21].

2.1.5. Conclusions. We were the first to construct a consistent physicomathematical model of propagation of electromagnetic waves in layered media without recourse to the matrices of the induced-surface-charge impedances. This model is based on the Maxwell equations, the electric-charge conservation law, the total-current continuity, and the Dirichlet theorem. Our numerical investigations have shown that the physical and mathematical model proposed can be used to advantage for simulation of the propagation of a highfrequency electromagnetic wave in a medium consisting of layers having different electrophysical properties.
2.2. Wave Equation for $\vec{H}$ and Conditions on the Boundaries in the Presence of Strong Discontinuities of the Electromagnetic Field. Numerical Modeling of Electrodynamic Processes in the Surface Layer

2.2.1. Introduction. During the interaction of an external magnetic field and magnetic abrasive particles, the particles are magnetized, and magnetic dipoles with the moment oriented predominantly along the field are formed. "Chains" along the force lines of the field $[22,23]$ appear that periodically act on the processable surface with a frequency $\omega=l / v$. A fixed elemental area of the material periodically experiences the effect of the magnetic field of one direction. Actually, the frequency and duration of the pulse will be still higher because of the rotation of the magnetic abrasive particle due to the presence of the moment of forces on contact and of the friction of the particle against the processable part. In what follows, we will not take into account the effect of rotation.

We assume that the particle velocity on the polisher is $v$. If the particle radius is $r$, then the angular frequency is $\omega=2 \pi v / r$, and precisely this frequency determines the frequency of the effect of the variable magnetic field component due to the fact that for a ferromagnetic $\mu>1$. The magnetic permeability $\mu$ of ferromagnetics, which are usually used in magnetoabrasive polishing, is measured by thousands of units in weak fields. However, in polishing, the constant external magnetic field is strong and amounts to 


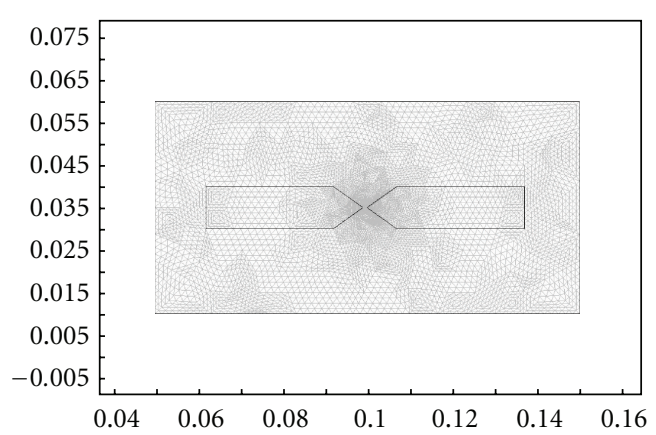

(a) Mesh

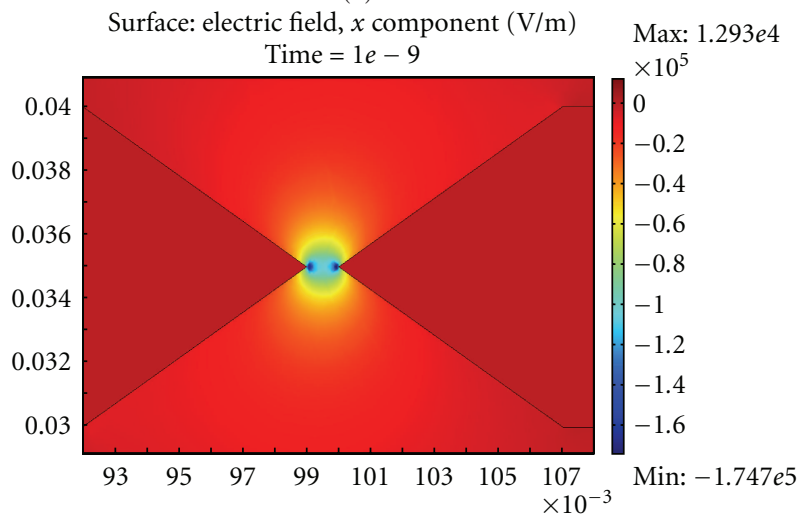

(c) Amplitude $E_{x}$

Surface: electric field, $y$ component $(\mathrm{V} / \mathrm{m})$

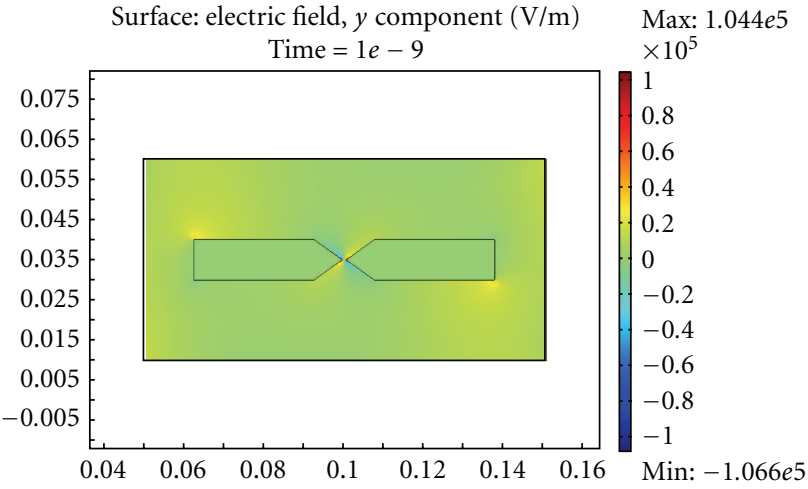

(e) Amplitude $E_{y}$

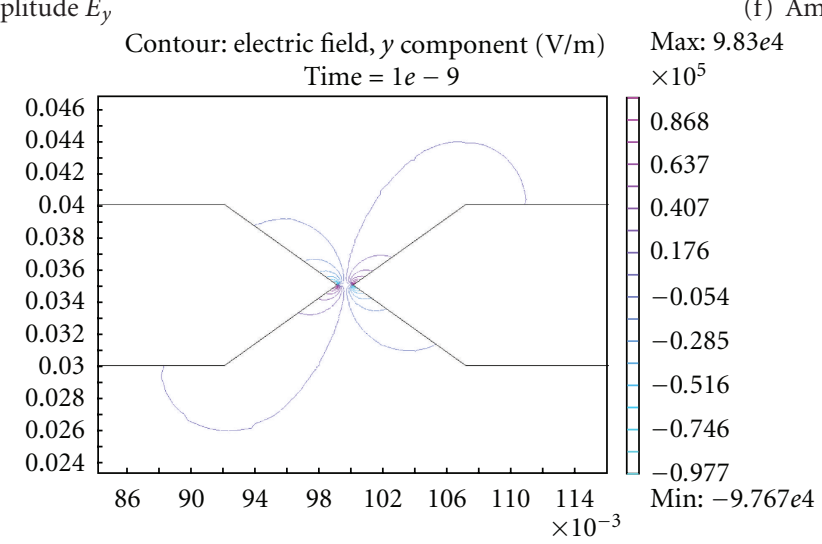

(g) Isolines $E_{y}$

FIGURE 7: Mesh, amplitude $E_{x}$ and isolines, and amplitude $E_{y}$ and isolines of the electromagnetic field strength.
Surface: electric field, $x$ component $(\mathrm{V} / \mathrm{m}) \quad$ Max: $1.293 e 4$

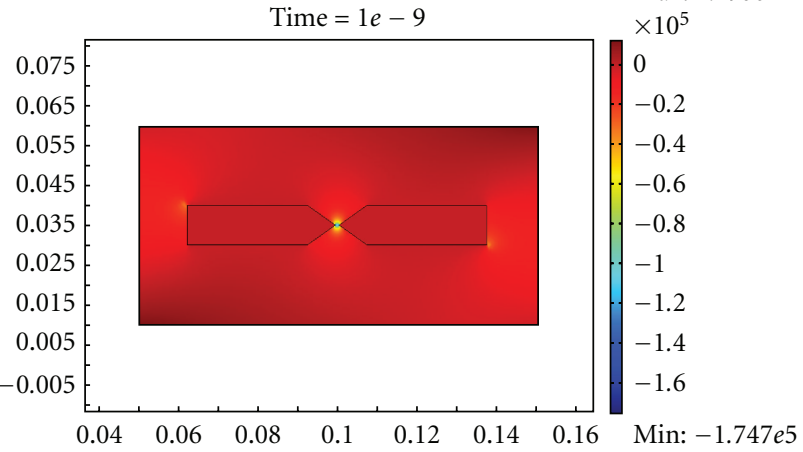

(b) Amplitude $E_{x}$

Contour: electric field, $x$ component $(\mathrm{V} / \mathrm{m}) \quad$ Max: 6908.465

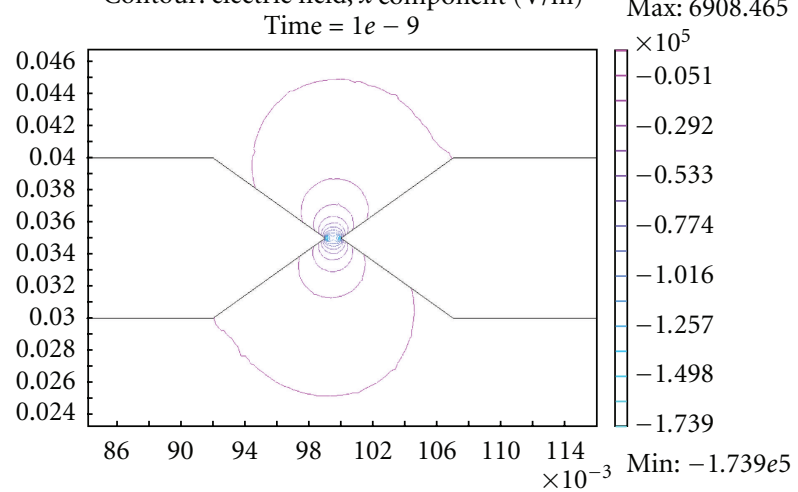

(d) Isolines $E_{x}$

Surface: electric field, $y$ component $(\mathrm{V} / \mathrm{m}) \quad$ Max: $1.044 e 5$

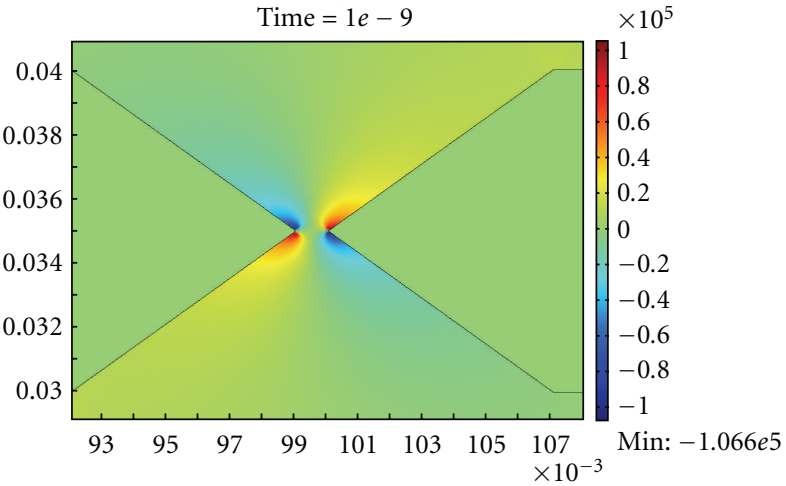

(f) Amplitude $E_{y}$

Max: $9.83 e 4$

$\times 10^{5}$ 


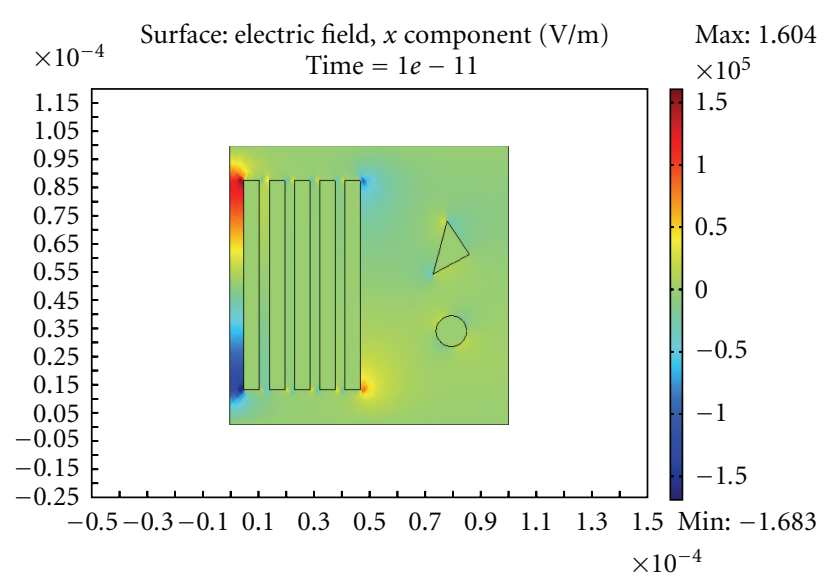

(a) Amplitude $E_{x}$

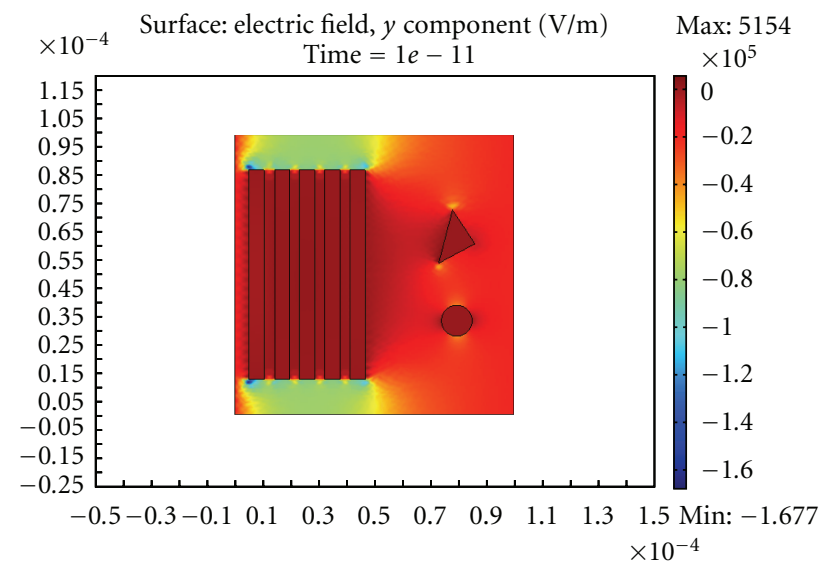

(c) Amplitude $E_{y}$

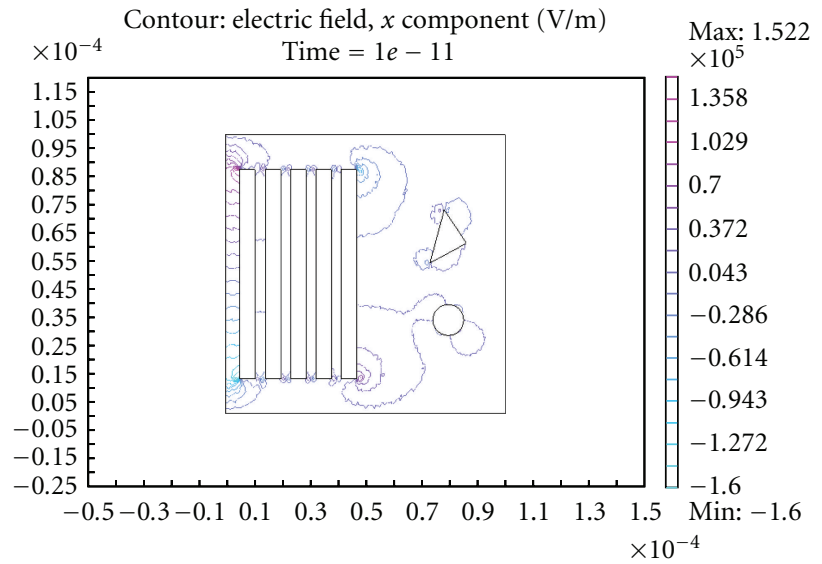

(b) Isolines $E_{x}$

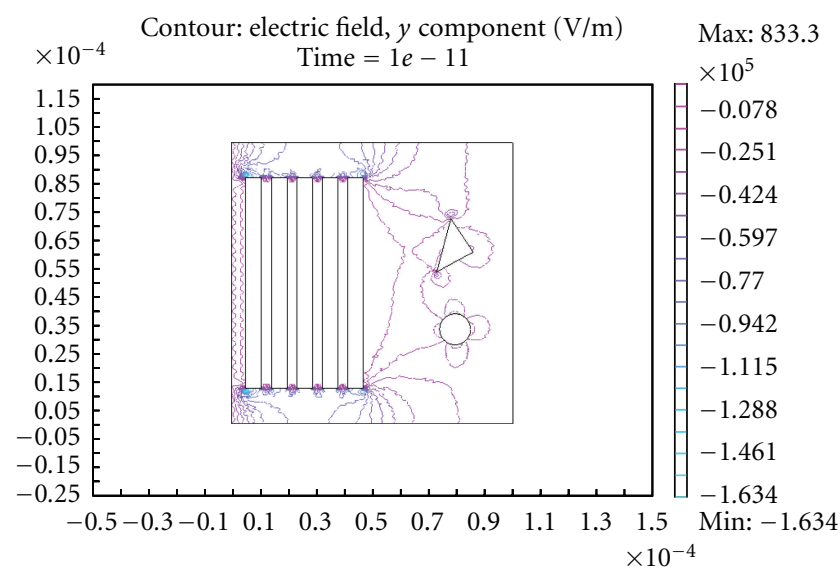

(d) Isolines $E_{y}$

Figure 8: Amplitude $E_{x}$ and isolines and amplitude $E_{y}$ and isolines of the electromagnetic field strength.

$10^{5}-10^{6} \mathrm{~A} / \mathrm{m}$, and in this case the value of $\mu$ for compounds of iron and nickel and for Heusler alloy decreases substantially.

Because of the presence of a strong external magnetic field $H_{0}$ the "small" absolute value of $\mu$ of an abrasive particle leads to a periodic "increase" and "decrease" in the normal component of the magnetic induction near the processable surface. In the present work we used neodymium magnets (neodymium-iron-boron) with $H_{0}>485,000 \mathrm{~A} / \mathrm{m}$. The magnetic permeability of a magnetic abrasive particle based on carbonyl iron was assumed in this case to be equal to $\mu_{1}=100$.

Due to the continuity of the normal magnetic induction component $B_{n 1}=B_{n 2}$, where $B_{n 1}=\mu_{1} \mu_{0} H_{1} ; B_{n 2}=\mu_{2} \mu_{0} H_{2}$. For example, in glasses $\left(\mu_{2}=1\right.$; therefore at the boundary of contact of the glass with the magnetic abrasive particle an additional variable magnetic field of strength $H_{1}>H_{0}$ appears.

In [24-27], magnetic field-induced effects in silicon are considered: a nonmonotonic change in the crystal lattice parameters in the surface layer of silicon, the gettering of defects on the surface, the change in the sorption properties of the silicon surface, and the change in the mobility of the edge dislocations and in the microhardness of silicon.

In [28-30], the influence of an electromagnetic field on the domain boundaries, plasticity, strengthening, and on the reduction of metals and alloys was established.

In view of the foregoing, it is of interest to find the relationship between the discrete-impulse actions of a magnetic field of one direction on the surface layer of the processable material that contains domains. According to [22], the size of domains is as follows: $0.05 \mu \mathrm{m}$ in iron, $1.5 \mu \mathrm{m}$ in barium ferrite; $8 \mu \mathrm{m}$ in the $\mathrm{MnBi}$ compound, and $0.5-$ $1 \mu \mathrm{m}$ in the acicular gamma ferric oxide. According to [31], the size of a domain may reach $10^{-6} \mathrm{~cm}^{3}$ (obtained by the method of magnetic metallography).

As a rule, an abrasive exhibits a distinct shape anisotropy, whereas the frequency of the effect is determined by the concentration of abrasive particles in a hydrophobic solution and by the velocity of its motion. We assume that on the surface of a processable crystal the magnetic field strength $H(t)=H_{1} \sin ^{4}(\omega t)+H_{0}$.

It is required to find the value of the magnetic field strength in the surface layer that has the characteristics $\lambda_{1}, \varepsilon_{1}$, 


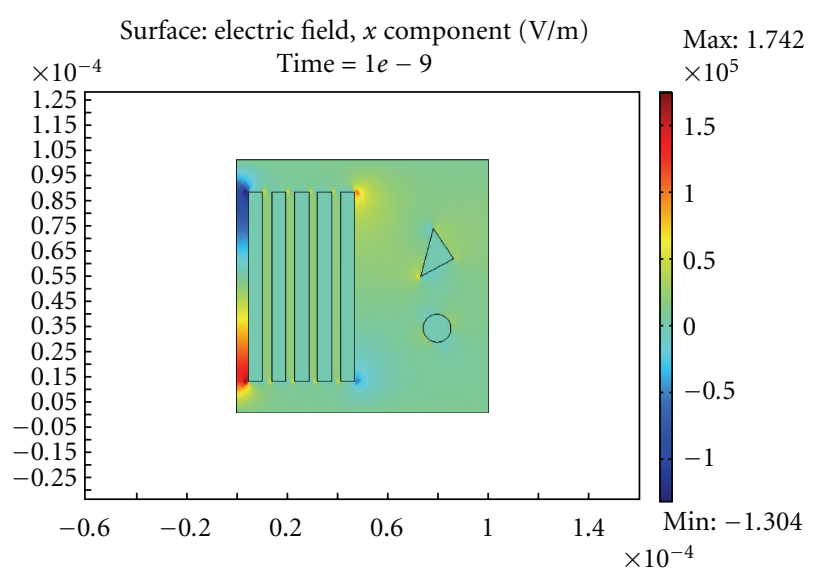

(a) Amplitude $E_{x}$

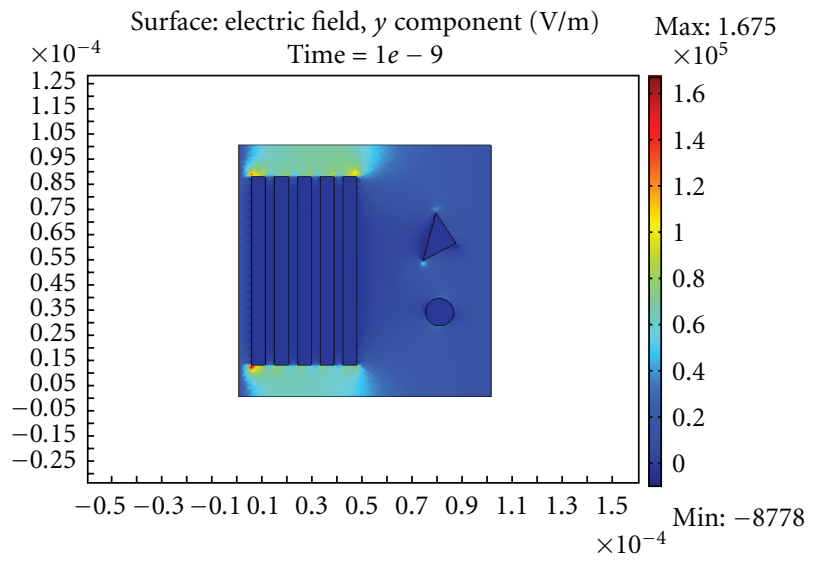

(c) Amplitude $E_{y}$

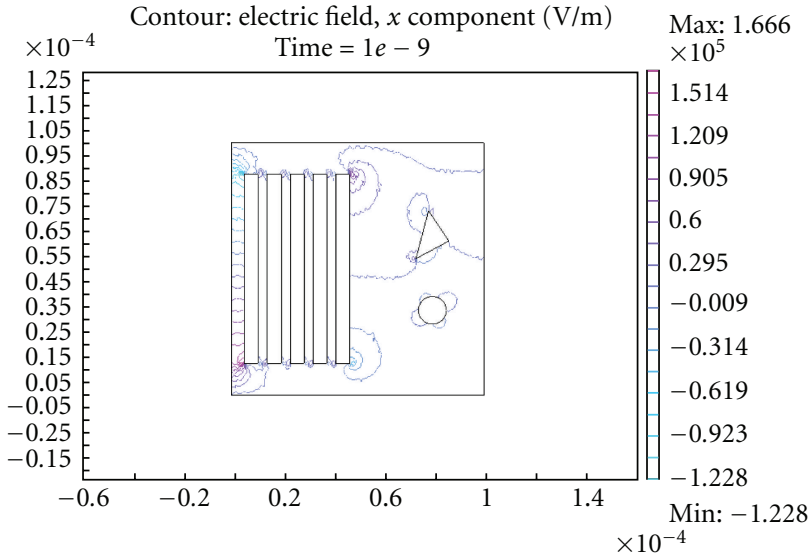

(b) Isolines $E_{x}$

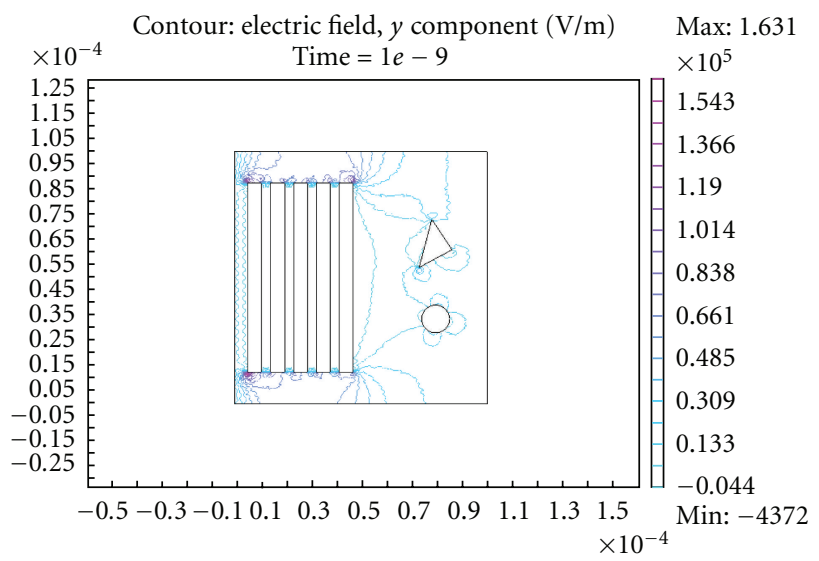

(d) Isolines $E_{y}$

Figure 9: Amplitude $E_{x}$ and isolines and amplitude $E_{y}$ and isolines of the electromagnetic field strength.

and $\mu_{1}$ and contains domains with electrophysical properties $\lambda_{2}, \varepsilon_{2}$, and $\mu_{2}$. The domains may have the form of a triangular prism, a bar, a cylinder, and so forth.

2.2.2. Physicomathematical Model: Wave Equation for $\vec{H}$. We consider interaction of a nonstationary magnetic field with substance on an example of a specific technology of magneto abrasive polishing based on use of magneto-rheological suspension. We will formulate a physicomathematical model of propagation of electromagnetic waves in a heterogeneous medium. The media in contact are considered homogeneous. We operate with the operator rot on the left- and righthand sides of the first equation for the total current (6) and multiply by $\mu_{0} \mu$; then we differentiate the second equation in (7) with respect to time. Taking into consideration the solenoidality of the magnetic field (7) and the rule of repeated application of the operator $\nabla$ to the vector $\overrightarrow{\mathbf{H}}$, we obtain

$$
\mu_{0} \varepsilon \varepsilon_{0} \frac{\partial^{2} \overrightarrow{\mathbf{H}}}{\partial t^{2}}+\lambda \mu_{0} \frac{\partial \overrightarrow{\mathbf{H}}}{\partial t}=\frac{1}{\mu} \nabla^{2} \overrightarrow{\mathbf{H}}
$$

In the Cartesian coordinates,(35) will have the form

$$
\begin{gathered}
\varepsilon \varepsilon_{0} \frac{\partial^{2} H_{x}}{\partial t^{2}}+\lambda \mu_{0} \frac{\partial H_{x}}{\partial t}=\frac{1}{\mu}\left(\frac{\partial^{2} H_{x}}{\partial x^{2}}+\frac{\partial^{2} H_{x}}{\partial y^{2}}+\frac{\partial^{2} H_{x}}{\partial z^{2}}\right), \\
\varepsilon \varepsilon_{0} \frac{\partial^{2} H_{y}}{\partial t^{2}}+\lambda \mu_{0} \frac{\partial H_{y}}{\partial t}=\frac{1}{\mu}\left(\frac{\partial^{2} H_{y}}{\partial x^{2}}+\frac{\partial^{2} H_{y}}{\partial y^{2}}+\frac{\partial^{2} H_{y}}{\partial z^{2}}\right), \\
\varepsilon \varepsilon_{0} \frac{\partial^{2} H_{z}}{\partial t^{2}}+\lambda \mu_{0} \frac{\partial H_{z}}{\partial t}=\frac{1}{\mu}\left(\frac{\partial^{2} H_{z}}{\partial x^{2}}+\frac{\partial^{2} H_{z}}{\partial y^{2}}+\frac{\partial^{2} H_{z}}{\partial z^{2}}\right) .
\end{gathered}
$$

One fundamental electromagnetic field equation is the equation $\operatorname{div} \vec{B}=0$. The use of the Dirichlet theorem for approximation of the value of the magnetic field strength on the boundaries between adjacent media analogously to that of the electric field strength does not necessarily guarantees the observance of the condition of solenoidality of the magnetic field; furthermore, the magnetic properties of heterogeneous media were assumed constant in deriving generalized wave equations. The experience of numerical calculations has shown that when it is necessary to model nonstationary magnetic phenomena it is better in many cases to use a generalized wave equation for $\vec{E}$, accordingly 
expressing $\vec{H}(t, \vec{r})$ by $\vec{E}(t, \vec{r})$ and, if need be, to perform backward recalculation to $\vec{H}(t, \vec{r})$. This approach is difficult to apply to modeling of heterogeneous media with different magnetic properties, when the magnetic permeability $\mu$ is dependent on coordinates.

In media with a weak heterogeneity where $\mu(x, y, z)$ is a piecewise continuous quantity, the application of the proposed method of through counting is quite justified. Indeed, the system of (13) and (36) yields that the function's discontinuity on the boundaries between adjacent media is determined by the complexes which will be called the generalized permeability $\varepsilon^{*} \equiv \varepsilon \mu \varepsilon_{0} \mu_{0}$ and the generalized conductivity $\lambda^{*} \equiv \lambda \mu \mu_{0}$. Using the Direchlet theorem for $\varepsilon^{*}$ and $\lambda^{*}$, we obtain their values on the boundaries between adjacent media and the values for the electric field strength at the discontinuity point (see (34)); here, we note that the value of the electric field strength is obtained without solving Maxwell equations. In fact, at the discontinuity point, we use linear interpolation of the function to obtain the values of $\varepsilon^{*}, \lambda^{*}$, and $E_{x=\xi}=(1 / 2)[E(\xi-0)+E(\xi+0)]$. Consequently, for piecewise continuous quantity $\mu(x, y, z)$, the application of the proposed method of through counting is justified. We note that the equality of the derivatives of the electric field strength along the normal to the surface at the discontinuity point according to Equation (32) holds. When the wave equation for $\vec{H}$ is used for media with different magnetic permeabilities the condition of equality of the derivatives fails, that is,

$$
\left.\frac{\partial H_{x}}{\partial x}\right|_{x=\xi-0} \neq\left.\frac{\partial H_{x}}{\partial x}\right|_{x=\xi+0},
$$

which is a consequence of (10); therefore, the use of throughcounting schemes for the wave equation for $\vec{H}$ is difficult.

Moreover, reformulation of the boundary conditions for equations (6) and (7), with electric field strength being eliminated from the boundary conditions, does not even allow prediction of the surface current direction. Indeed, magnetic field strength $\vec{H}$ is an axial vector which on substitution $\vec{r} \rightarrow(-\vec{r})$ must remain constant. The value of the surface current also cannot be determined without knowledge of the polar vector of electric field strength. The generalized wave equation for $\vec{E}$ contains the term grad $\operatorname{div} \vec{E}$ which directly allows for the influence of induced surface charges on the propagation of waves, the right-hand side of the generalized wave equation has the same form as the equations of the linear elasticity theory, hydrodynamics. We note that the proposed method of calculation can be used on condition that there are no built-in space charges and extraneous electromotive forces [13].

By virtue of what has been stated above, for modeling of the propagation of electromagnetic waves in glasses having roughness and defects, we used system (13) with boundary conditions (22)-(31) and (32).

2.2.3. Results of Numerical Simulation. The physicomathematical model developed can also efficiently be used in modeling the propagation of electromagnetic waves in media with complex geometries and strong electromagnetic field discontinuities.

The transverse cut of a cellular structure represents a set of parallelepipeds and triangular prisms of various cross-sections, as depicted in Figures 10(a) and 11(a). An electromagnetic wave propagates across the direction of parallelepipeds and triangular prisms (channels) along the coordinate $x$.

The size of the investigated two-dimensional object is $14 \times 20 \cdot 10^{-6} \mathrm{~m}$, and the sizes of the domains are $2-4 \mu \mathrm{m}$. The frequency of the influence of the magnetic field is $\omega=$ $2 \pi \cdot 10^{6}$, and the strength of the field is

$$
H_{x}=21 \cdot 10^{5} \sin ^{4}\left(2 \pi \cdot 10^{6} t\right) \frac{\mathrm{A}}{\mathrm{m}} .
$$

The electrophysical properties are as follows: of the large parallelepiped, $\mu=1, \varepsilon=8, \sigma=10^{-9} \Omega \cdot \mathrm{m}$; of domains, $\mu=1, \varepsilon=6, \sigma=10^{-8} \Omega \cdot \mathrm{m}$. They correspond to the electrophysical properties of glasses.

It was assumed that in a layer of thickness $15-20 \mu \mathrm{m}$ an electromagnetic wave propagates without attenuation; therefore, on all the faces of the large parallelepiped the fulfillment of condition (38) was considered valid. On the faces of the parallelepiped that are parallel to the $O X$ axis condition (38) corresponded to the "transverse" tangential component of the wave; on the faces parallel to $O Y$ condition (38) corresponded to the normal component of the field.

The calculations were carried out with a time step of $10^{-13} \mathrm{sec}$ up to a time instant of $10^{-10} \mathrm{sec}$.

Figures 10(a) and 11(a) present the amplitude values of the magnetic field strength along $H_{x}$ and $H_{y}$ with a comparison scale, whereas Figures 11(b) and 11(b) present the corresponding isolines. An analysis of these figures shows that at the places of discontinuity, on the wedges, force lines of the electromagnetic field concentrate. According to [32], precisely wedges are often the sources and sinks of the vacancies that determine, for example, the hardness and plasticity of a solid body.

Also, we modeled the propagation of waves in media, when domains possess magnetic properties. We assumed, in the calculations, that $\mu=100$; the remaining parameters correspond to the previous example of solution (Figure 12).

Of interest is the interaction of the electromagnetic wave with the rough surface shown in Figures 13 and 14. As in the previous examples, we observe the concentration of electromagnetic energy on angular structures.

From Figures 13 and 14, it is seen that electromagnetic heating of tapered structures may occur in addition to mechanical heating in magnetic abrasive machining.

As we have mentioned above, for investigation of the propagation of electromagnetic waves in nonmagnetic materials, it is more expedient to use the generalized equation for $\vec{E}$. For the purpose of illustration we give an example of numerical calculation of an optical knife with the wave equation for $\vec{H}$ (Figure 15).

From Figure 15, it is seen that the actual problem of diffraction on the optical knife remains to be solved, that is, there is no "glow" on the optical-knife section, which is inconsistent with experimental data. 


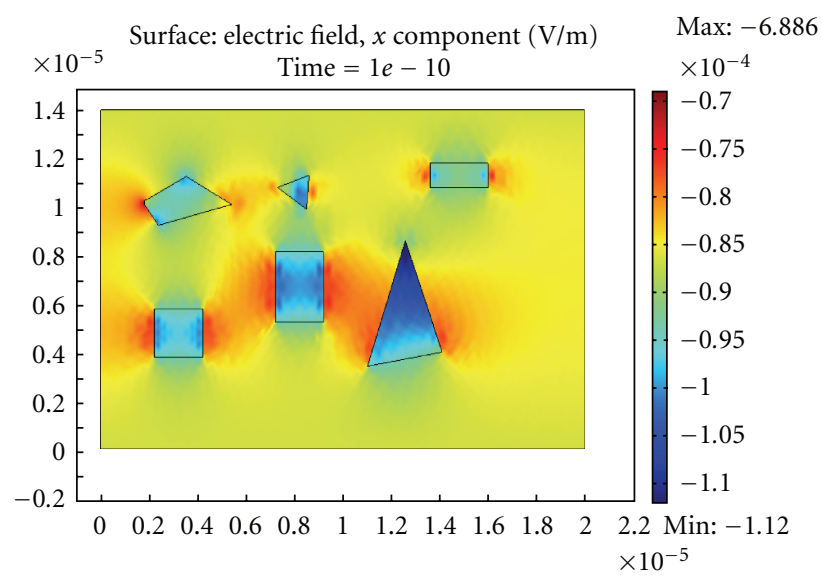

(a) Amplitude $H_{x}$

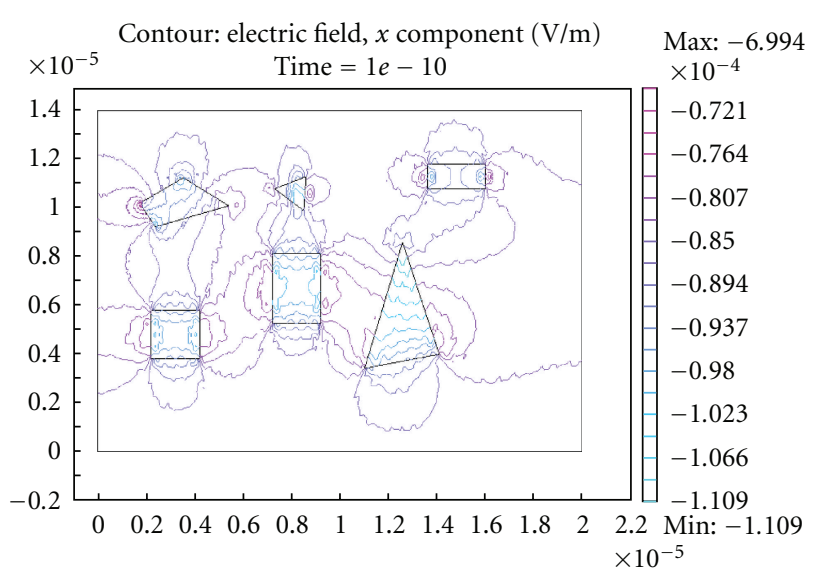

(b) Isolines

FIgURE 10: Amplitude $H_{x}$ and isolines of the magnetic field strength.

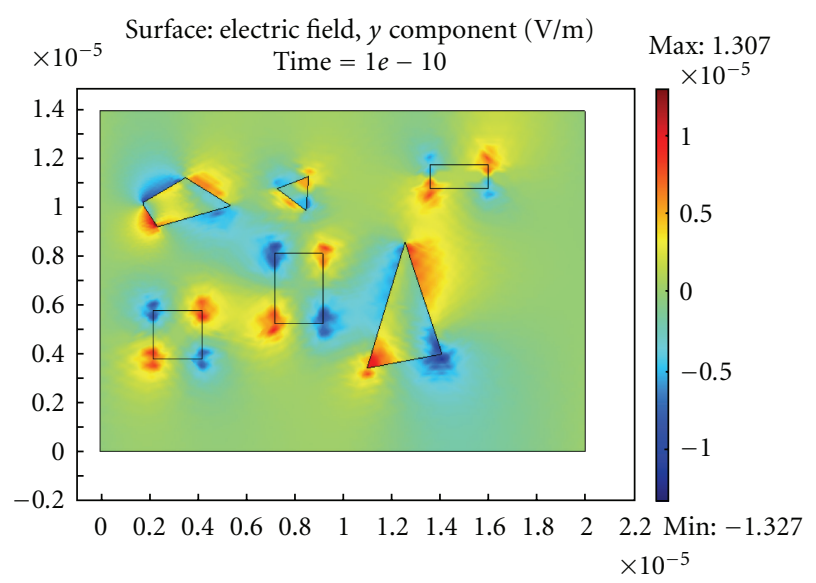

(a) Amplitude $H_{y}$

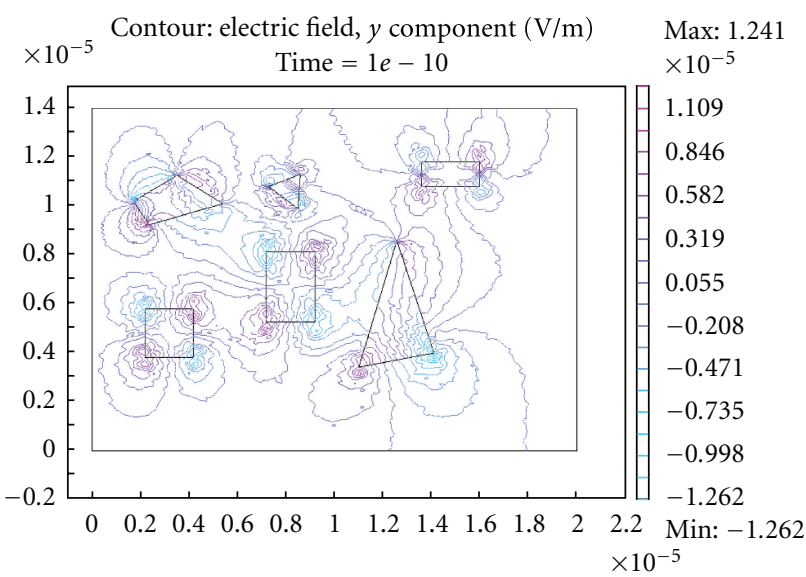

(b) Isolines

FIgURE 11: Amplitude $H_{y}$ and isolines of the magnetic field strength.

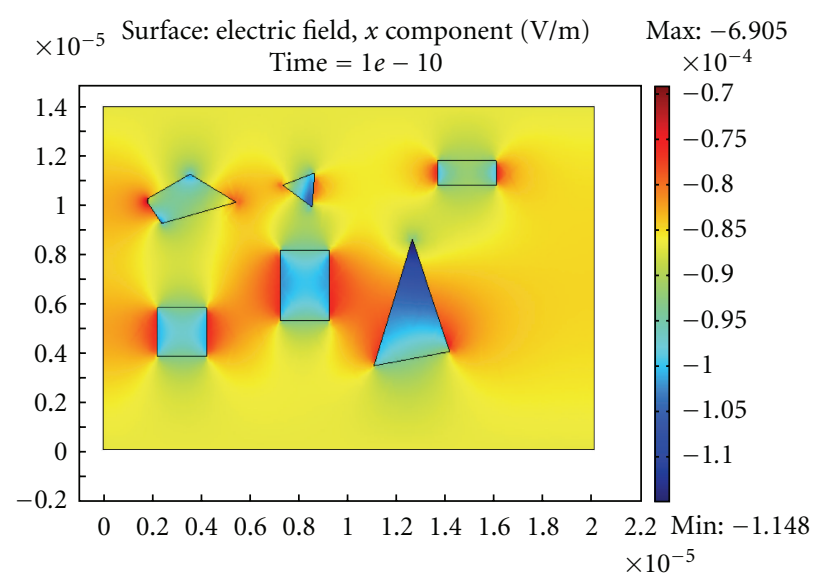

(a) Amplitude $H_{x}$

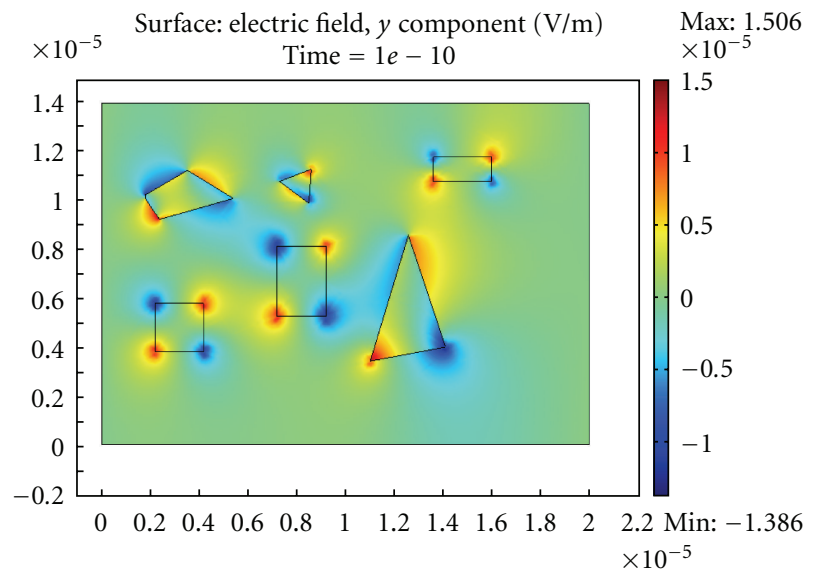

(b) Amplitude $H_{y}$

FIGURE 12: Amplitude $H_{x}$ and $H_{y}$ of the magnetic field strength. 


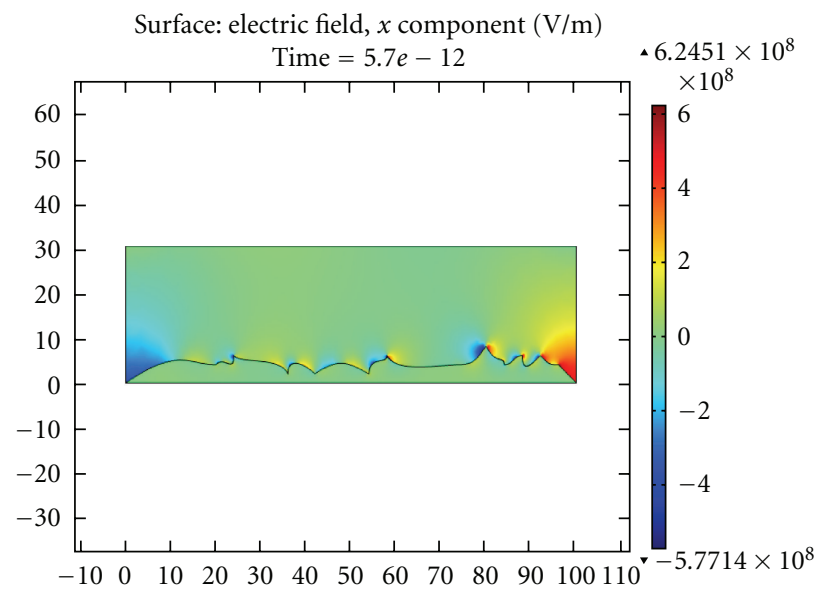

(a) Amplitude $H_{x}$

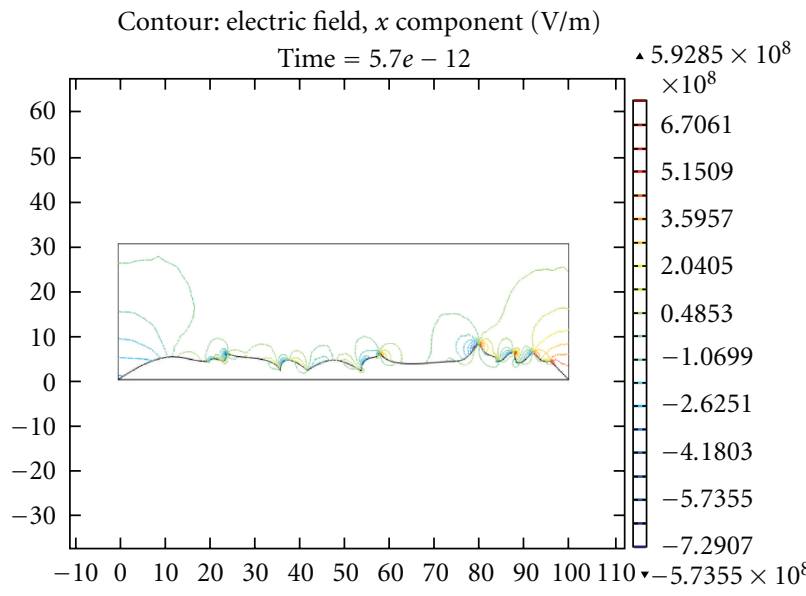

(b) Isolines

FIGURE 13: Amplitude $H_{x}$ and isolines of the magnetic field strength.

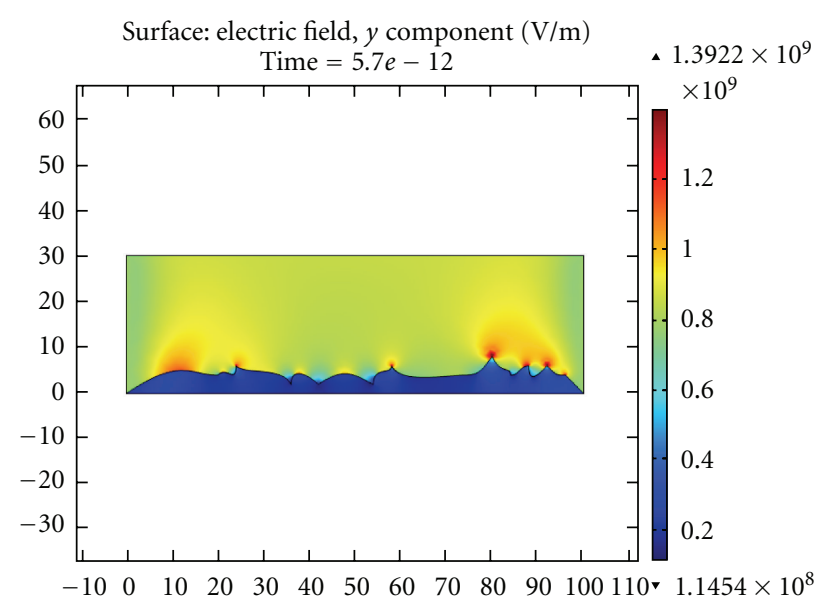

(a) Amplitude $H_{x}$

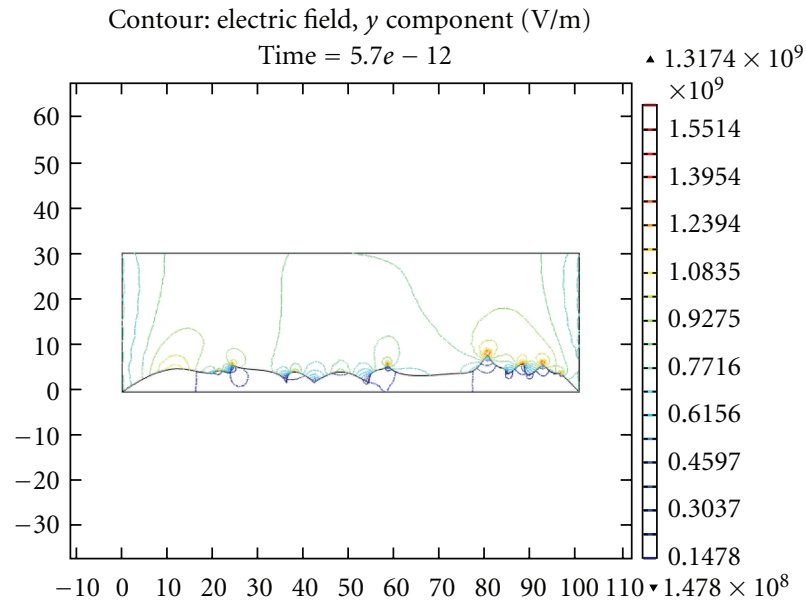

(b) Isolines

Figure 14: Amplitude $H_{y}$ and isolines of the magnetic field strength.

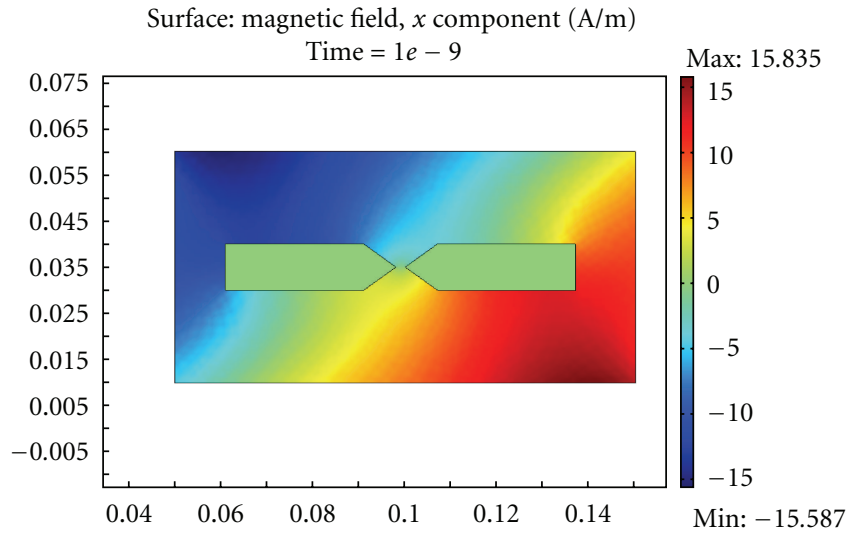

(a) Amplitude $H_{x}$

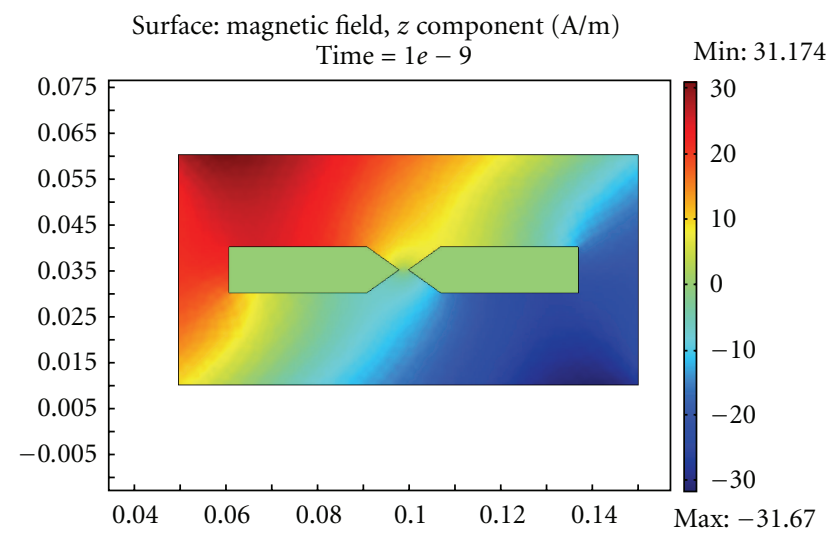

(b) Amplitude $H_{y}$

Figure 15: Amplitude $H_{x}$ and $H_{y}$ of the magnetic field strength. 
As is known [33] in thermodynamically equilibrium systems the temperature $T$ and the electrical $\varphi$ and chemical $\mu_{c}$ potentials are constant along the entire system:

$$
\operatorname{grad} T=0, \quad \operatorname{grad} \varphi=0, \quad \operatorname{grad} \mu_{c}=0 .
$$

If these conditions are not fulfilled $(\operatorname{grad} T \neq 0$, grad $\varphi \neq 0$, grad $\mu_{c} \neq 0$ ), irreversible processes of the transfer of mass, energy, electrical charge, and so forth appear in the system.

The chemical potential of the $j$ th component is determined, for example, as a change of the free energy with a change in the number of moles:

$$
\mu_{c j}=\left(\frac{\partial F}{\partial n_{j}}\right)_{T, V},
$$

where

$$
d F=-S d T-P d V+\vec{H} d \vec{B}+E d D .
$$

The last term in (41) takes into account the change in the free energy of a dielectric due to the change in the magnetic induction. The free energy of a unit volume of the dielectric in the magnetic field in this case has the form

$$
F(T, D)=F_{0}+\mu \mu_{0} \frac{\overrightarrow{H^{2}}}{2}+\varepsilon \varepsilon_{0} \frac{E^{2}}{2} .
$$

We assume that changes in the temperature and volume of the dielectric are small. Then the mass flux is determined by a quantity proportional to the gradient of the chemical potential or, according to (41), we obtain

$$
q_{i}=-D_{\mu_{c}} \operatorname{grad}(\vec{H} d \vec{B}+E d D)=-D_{\mu_{c}} \operatorname{grad} W,
$$

where $W=\mu \mu_{0}\left(\vec{H}^{2} / 2\right)+\varepsilon \varepsilon_{0}\left(E^{2} / 2\right)$ is the density of the magnetic field in the unit volume of the dielectric.

In magnetic abrasive polishing on the sharp protrusions of domains the gradients of magnetic energy are great, which can lead to the origination of vacancy flows.

An analysis of the results shows that the nonstationary component of the full electromagnetic energy is also concentrated in the region of fractures and wedges, that is, at the sharp angles of domains, which may lead to the improvement of the structure of the sublayer of the treated surface due to the "micromagnetoplastic" effect. Maximum values of the nonstationary part of the total electromagnetic energy $W_{\max }$ in the sublayer corresponds to a maximum value of the function $\sin \left(2 \pi \cdot 10^{6} t\right)$ and occur for the time instants $t=$ $(n / 4) 10^{-6}$ sec, where $n$ is the integer, with the value of $W_{\max }$ for a neodymium magnet and a magnetoabrasive particle on the basis of carboxyl iron amounting to a value of the order of ((5)-(6)) $110^{6} \mathrm{~J} / \mathrm{m}^{3}$. Having multiplied $W_{\max }$ by the volume of a domain, vacancy, or atom, we may approximately obtain the corresponding energy. The density of the electromagnetic energy in all of the cases is much smaller than the bonding energy of atoms, $10^{-18}-10^{-19} \mathrm{~J}$. However, a periodic change in the magnetic field in one direction leads to a ponderomotive force that may influence the motion of various defects and dislocations to create a stable and equilibrium structure of atoms and molecules in magnetic abrasive polishing and, on the long run, in obtaining a surface with improved characteristics due to the "micromagnetoplastic" effect. The result of Para 2.2 were published in part [34].

\section{Ion Conductors: New Closing Relations on the Boundaries of Adjacent Media}

3.1. Diffusion Electric Phenomena in Electrolytes. The medium under discussion is assumed to consist of a non ionized solvent, an electrolyte in the form of ions and uncharged components. At first we consider infinitely diluted electrolytes when molecules are completely dissociated into cations and anions.

In a solution, ions are transferred by convection, diffusion, and migration in the presence of an electric field. Derivation of the equation for ion migration is based on the following considerations. In a solution with molar concentration $n$ and diffusion coefficient $D_{i}$ let there be ions with charge $z_{i}$. When an ion is exposed to an electric field with intensity $E$ applied to the solution, the ion experiences the force $z_{i} e \mathbf{E}$, which brings it into motion. The ion velocity is related to this force by the usual expression known for the motion of particles in a viscous medium:

$$
u=\gamma_{i}^{*} z_{i} e \mathbf{E},
$$

where $\gamma_{i}^{*}$ is the ion mobility. The latter may be expressed in terms of the diffusion coefficient using the known Einstein relation:

$$
\gamma_{i}^{*}=\frac{D_{i} F}{R T} .
$$

The total flux of ions of the $i$ th kind in a moving medium in the presence of diffusion and migration is determined by the Nernst-Planck equation:

$$
q_{i}=n_{i} v-D_{i} \nabla n_{i}+\frac{D_{i} z_{i} F \mathbf{E}}{R T} n_{i} .
$$

Formulas (44) and (45) have, in fact, a limited sphere of applicability. Indeed, Einstein's work [35] is concerned only with the diffusion of a neutral impurity with its small concentration in a solution when the usual relations of hydrodynamics are valid for a flow around a sphere.

In the physics of plasma, formulas (44) and (45) are based on other considerations and provided the plasma is weakly ionized, that is, particles move independently of each other. Here, only the collision of charged particles with neutral ones is taken into account [36].

In $[37,38]$, for describing the diffusion and migration of ions in a partially dissociated electrolyte it is suggested to take into consideration their transfer by neutral molecules. In the theory developed by Y. Kharkats [37] the expression for the total flux of ions is

$$
q_{i}=n_{i} v-D_{i} \nabla n_{i}+\frac{D_{i} z_{i} F \mathbf{E}}{R T} n_{i}-D z_{i} \nabla n_{A} .
$$


TABLE 1: The degree of $\mathrm{HCl}$ electrolytical dissociation in terms of measured electric conductivity $\alpha_{1}$ and E.m.f. $\alpha_{2}$.

\begin{tabular}{lcccccc}
\hline$C_{\mathrm{HCl}}$, mole/liter & 0.003 & 0.08 & 0.3 & 3.0 & 6.0 & 16.0 \\
\hline$\alpha_{1}$ & 0.986 & 0.957 & 0.903 & - & - & - \\
$\alpha_{2}$ & 0.99 & 0.88 & 0.773 & 1.402 & 3.4 & 13.2 \\
\hline
\end{tabular}

The last term in (47) takes into account ion transfer by a flux of neutral molecules; here the concentrations of anions, cations, and neutral dissociated molecules are determined by the chemical equilibrium conditions

$$
\beta=\frac{\left(n^{-}\right)^{z^{+}}\left(n^{+}\right)^{z^{-}}}{n_{A}}
$$

where $\beta$ is the dissociation equilibrium constant. If the degree of dissociation at the prescribed total concentration $n$ is $\alpha$, then $n_{A}=(1-\alpha) n$ and, consequently,

$$
\beta=\frac{\alpha^{2} n}{1-\alpha}
$$

The dissociation constant $\beta$, unlike the degree of dissociation, must not depend on concentration. However, in real partially dissociated electrolytes those arguments for $\alpha$ and $\beta$ are in rather poor agreement with experiment. In [39] the dissociation degree for $\mathrm{HCl}$ is listed in Table 1 as a function of concentrations, calculated in terms of measured electric conductivity $\alpha_{1}$ and e.m.f. $\alpha_{2}$.

Inspection of the table reveals that the dissociation degrees obtained by different experimental methods coincide best in the case of dilute solutions. In the high-concentration range of the electrolyte, $\alpha_{2}$ even exceeds unity, which, naturally, has no physical sense.

According to the Arrhenius theory the dissociation constant $\beta$ for the given electrolyte at the prescribed temperature and pressure must remain constant independently of the solution concentration. In [40], the dissociation constants of some electrolytes are given at their different concentrations. Only for very weak electrolytes (solutions of ammonia and acetic acid) does the dissociation constant remain more or less constant on dilution. For strong electrolytes (potassium chloride and magnesium sulfate), it changes by severalfold and in no way may be considered a constant.

Of course, one may formally take into account the dependence of the dissociation constant on the electrolyte concentration and use it in the modified Nernst-Planck equation (47) and (48) but the main drawback in describing separately the diffusion and migration of ions in an electrolyte, in D. I. Mendeleev's opinion, lies in the fact that the interaction of particles of a dissolved substance between each other as well as with solvent molecules is neglected. Also, he has pointed out that not just the processes of formation of new compounds with solvent molecules are of importance for solutions. D. I. Mendeleev's viewpoints have been extended by Sakhanov [40], who believed that in addition to the usual dissociation reaction in an electrolyte solution there also proceeds associating of simple molecules. Molecular associations dissociate, in their turn, into complex and simple ions. In this case (47) will not hold mainly because of the fact that the expressions for diffusion, migration, and nondissociated flows of molecules must be determined relative to some mean liquid velocity. In concentrated solutions, this velocity does not coincide with the solvent velocity and must be determined from the fluid dynamics equations of a multicomponent mixture in which characteristics of its components (physical density, charge, diffusion coefficient of a complex ion) are, in fact, unknown. This is a reason why the theory of diffusion and migration of ions for a partially dissociated electrolyte solution, with a current traversing through it, encounters crucial difficulties. Besides, it is rather difficult to take an account of the force interaction of complex cations and anions between each other and with an external electric field. Therefore Einstein's formula (44) will also change its form.

The main drawbacks of the theory of electrolytic dissociation are fully defined in collected papers "Fundamental Principles of Chemistry" by D. I. Mendeleev as well as in $[40,41]$.

In our opinion, the principal disadvantage of the approaches considered above lies in the fact that the NernstPlanck equation as well as its modified form for a partially dissociated electrolyte (see (47)) are based on the hydrodynamic theory of diffusion. We shall substantiate below that it is more reasonable to use the equations of thermodynamics of irreversible processes.

When applying the Nernst-Planck equation (see (46)) and its modified forms (see (47)), it is implicitly postulated that cations and anions in the electrolyte solution are different components. The equations of transfer of cations and anions are written separately. On the other hand, the commonly used equation of electroneutrality does not ensure, according to Gibbs [39], the independence of differentials of cations $\partial n_{i}$ and anions $\partial n_{e}$ in a volumetric electrolyte solution their changes are functionally related and therefore they are identical, indiscernible components.

3.1.1. The Case of a Current Traversing a Binary Fully Dissociated Electrolyte. Let us write system of equations (see (46)) in the form:

$$
\begin{gathered}
\frac{\partial n_{e}}{\partial \tau}+\mathbf{v g r a d} n_{e}=D_{e} \Delta n_{e}+\frac{z_{e} F D_{e}}{R T} \operatorname{div}\left(n_{e} \mathbf{E}\right), \\
\frac{\partial n_{i}}{\partial \tau}+\mathbf{v g r a d} n_{i}=D_{i} \Delta n_{i}+\frac{z_{i} F D_{i}}{R T} \operatorname{div}\left(n_{i} \mathbf{E}\right), \\
z_{e} n_{e}-z_{i} n_{i}=0 .
\end{gathered}
$$

Analogously to [39], instead of concentrations $n_{e}$ and $n_{i}$ we introduce the molar concentration related to $n_{e}$ and $n_{i}$ by the expressions

$$
C=\frac{n_{e}}{z_{i}}=\frac{n_{i}}{z_{e}}
$$

Expressing $n_{e}$ and $n_{i}$ in (50) in terms of the molar concentration, we arrive at the following expression for the function C:

$$
\frac{\partial C}{\partial \tau}+v \nabla C=D_{\mathrm{ef}} \Delta C
$$


where

$$
D_{\mathrm{ef}}=\frac{D_{e} D_{i}\left(z_{e}+z_{i}\right)}{z_{e} D_{e}+z_{i} D_{i}}
$$

is the diffusion coefficient of a salt or the effective diffusion coefficient of a binary electrolyte. Expressing the concentrations $n_{e}$ and $n_{i}$ in terms of the molar concentration $C$ we find the expression for the vector of current density $\mathbf{J}$ [42]:

$$
\mathbf{J}=\left(D_{i}-D_{e}\right) F z_{i} z_{e} \nabla C+\frac{F^{2} z_{i} z_{e}}{R T}\left(z_{i} D_{i}+z_{e} D_{e}\right) C E
$$

In [43] it is shown that by its meaning $D_{\mathrm{ef}}$ is a coefficient of molecular diffusion. For instance, the diffusion coefficients of copper sulfate have an intermediate value between the diffusion coefficients of copper and sulfate ions; if $D_{e}=$ $0.173 \cdot 10^{-9} \mathrm{~m}^{2} / \mathrm{sec}$ and $D_{i}=1.065 \cdot 10^{-9} \mathrm{~m}^{2} / \mathrm{sec}$, then $D_{\mathrm{ef}}=$ $0.854 \cdot 10^{-9} \mathrm{~m}^{2} / \mathrm{sec}$. However, expression (54) does not offer an explanation of the abnormally high mobility of $\mathrm{H}$ and $\mathrm{OH}$ ions when their displacement proceeds by a croquet-like mechanism [44].

In the system of (53)-(55) no symmetry of cross terms is observed; therefore, it is difficult to take into account "the superposition effects." Indeed, from (55) it is evident that the molar concentration gradients VC exert an influence on the current J. On the other hand, one can see from (53) that an electric field has no effect on the diffusion of the molecules, and therefore the Onsager reciprocal relation is disturbed.

One may raise the objection that according to the NernstPlanck equations it follows that the concentration gradients of cations and anions exert an influence on the potential distribution of an electric field [42]:

$$
\nabla\left(\rho_{R} \nabla \varphi\right)=-F \sum_{i} z \nabla\left(D_{i} \nabla C_{i}\right)
$$

However, relation (56) itself has been derived by Neumann [43] on the assumption that the resultant current does not depend on the mass flow.

On derivation of the equations for diffusion and migration of ions in a volumetric electrolyte solution we shall employ the relations of the thermodynamics of irreversible processes.

Finally, it is worthy of note that in plasma physics there is also no symmetry of "the superposition effects" in describing the ambipolar diffusion of ions, and the theory is based on the hydrodynamic model [36].

In investigating the dynamics of an electron-hole semiconductor plasma, the hydrodynamic model (50)-(51) is also used.

In our opinion, electric field-induced transfer of charges entails transfer of their kinetic energy as well as of heat and mass, and, conversely, mass or heat transfer may simultaneously cause, if we are concerned with the system of charged particles, charge transfer and give rise to an electromotive force.

3.1.2. Conclusion. To phenomenologically describe diffusional-electrical phenomena in electrolytes it is suggested to use the methods of the thermodynamics of irreversible processes, rather than the hydrodynamic theory of diffusion of ions, for the experimentally observable thermodynamic flows and forces without clear discernment and identification of real ion flows and mobilities.

3.2. Landau Model. In what follows we consider ion conductors that contain the electrolytic plasma with a fairly high concentration of charged particles. The interaction of the charged plasma particles with the external electromagnetic field and their collective interaction can theoretically be investigated completely only on the basis of the Boltzmann kinetic equation with a self-consistent field. In this probabilistic approach to the phenomena under study, one introduces characteristics that are average over a large ensemble of particles and are always related to the introduction of additional hypotheses on the properties of particles and their interactions and to the simplification of these properties and interactions. We note that in many cases, for example, when the electrolytic plasma in liquid electrolytes is considered, there is even no basis for constructing such methods. The developed methods are usually not an efficient means of solving problems by virtue of the excessive complexity of corresponding equations.

Another general method is construction of a phenomenological macroscopic theory based on the general regularities and hypotheses obtained experimentally; this precisely method will be developed in the present work. For many problems of applied character, consideration of a plasma as a conducting gas is sufficient. Such approximation is strictly justified only in the case of a dense plasma when the free path of charged particles is much smaller than the characteristic dimension of the system and particle collisions are of crucial importance. The velocity distribution of particles is Maxwellian; at each point, it is fully determined by the local values of density, temperature, and macroscopic velocity. With these conditions being observed, we can carry out, in a unified context, macroscopic phenomenological description of a gas medium, a metal and dielectric plasma, a plasma in a liquid electrolyte, or an electrolytic plasma.

The necessary condition of existence of the state of local thermodynamic equilibrium (LTE) of a plasma is high frequency of collisions of plasma particles (Maxwellization) so that the plasma state undergoes no marked changes over the period between collisions on the mean free path. The observance of this condition means the following: (1) the electrons have time to transfer the energy received from the electrostatic field to heavy particles; (2) ionization processes are nearly totally counterbalanced by recombination (the Saha equation holds true); (3) the overwhelming part of excited atoms gives up its energy in collisions; (4) energy exchange between particles dominates the processes in which the plasma energy markedly grows or diminishes.

In the LTE plasma state, it is only the radiation field (photons) that is not in equilibrium with the plasma, but the energy loss by radiation is minor in a number of cases compared to other mechanisms of energy transfer. With allowance for the assumptions enumerated above, 
the plasma electrodynamics can be described by Maxwell continuity, motion, and energy equations [45]. For macroscopic description of transfer processes in ion conductors, we will use the equations of thermodynamics of irreversible processes without separating explicitly ion carriers.

The possibility of such description was first suggested by L. D. Landau and E. M. Lifshits. This approach which has been substantiated above does not provide for the introduction and determination of the coefficients of molecular diffusion of cations and anions and of the degree of dissociation in an ion conductor. It is conditioned by the interaction of the mass and charge fluxes. The ion fluxes are not determined. The density of the conduction current $\mathbf{J}_{q}$, the mass flux $\mathbf{J}_{m}$, and the heat flux $\mathbf{J}_{T}$ for the medium's nonequilibrium state has the form [45]:

$$
\begin{aligned}
\mathbf{J}_{q} & =\lambda(\mathbf{E}-\beta \operatorname{grad} n)-\lambda \alpha(T) \operatorname{grad} T, \\
\mathbf{J}_{m} & =-D_{m} \operatorname{grad} n-D_{A}^{*} \lambda \mathbf{E}-D_{T} \operatorname{grad} T, \\
\mathbf{J}_{T} & =-k \operatorname{grad} T-I_{q}(\Pi+\varphi)-\lambda D_{T}^{*} \operatorname{grad} n,
\end{aligned}
$$

where $D_{M}$ is the coefficient of molecular diffusion, $D_{A}^{*}$ is the coefficient of ambipolar diffusion, $D_{T}^{*}$ is the coefficient allowing for heat transfer by the impurity motion, $\beta$ is the coefficient of specific electrical ambipolar conductivity due to $\nabla n$, and $\Pi$ is the Peltier coefficient.

To investigate the interaction of electromagnetic impotential fields (rot $\vec{E} \neq 0$ ) and heat and mass transfer rates it is advisable to use the system of (57) for polar vectors; if needed, the pseudovector of the magnetic field strength can be calculated from the Maxwell equation (7).

\subsection{Interaction of Nonstationary Electric, Thermal, and Diffu- sion Fields with Allowance for Mass Transfer in a Layered Medium with the Example of an Electrochemical Cell}

3.3.1. Introduction. Investigation of the interaction of electric and thermal fields with allowance for mass transfer and contact phenomena is a complex and topical problem of the theory and practice of various fields of natural science and technology.

The work seeks to construct a physicomathematical model of the interaction of nonstationary electric fields in a layered medium with allowance for nonstationary thermal phenomena and mass transfer without explicit separation of charge carriers. The media in contact are considered to be homogeneous. For the sake of clear representation, we will consider two-layer one-dimensional models.

3.3.2. Interaction of Electric and Thermal Fields. In different substances, the processes of transfer of charge and energy are interrelated. The quantity of the released heat is determined by Joule heating and by the effects of Thomson and Peltier. The problem of interaction of nonstationary thermal phenomena has been considered in [46] without taking into account the Thomson effect.

According to [45], the expressions for the conductioncurrent density and the energy-flux density in the absence of an external magnetic field or in the case of its slight influence have, respectively, the form

$$
\begin{aligned}
& I_{q}=-\lambda(\alpha(T) \operatorname{grad} T+\operatorname{grad} \varphi), \\
& I_{T}=-k(T) \operatorname{grad} T+I_{q}(\Pi+\varphi),
\end{aligned}
$$

where $a(T)$ is the specific thermoelectromotive force, $\Pi=\alpha T$ is the Peltier coefficient, $\varphi$ is the potential, and $k(T)$ is the thermal-conductivity coefficient. We note that the problem is always nonlinear.

Having eliminated the magnetic-field strength from system (13) according to one method of [47-49], we obtain the equation for the electric-field strength

$$
\frac{\varepsilon}{c^{2}} \frac{\partial^{2} \mathbf{E}}{\partial t^{2}}+\mu_{0} \frac{\partial \mathbf{I}_{q}}{\partial t}=\frac{1}{\mu} \nabla^{2} \mathbf{E} .
$$

The heat-balance equation has the form [45]

$$
\begin{aligned}
c_{p} \rho \frac{\partial T}{\partial t}= & \operatorname{div}(k(T) \operatorname{grad} T) \\
& +\mathbf{I}_{q} \cdot(\mathbf{E}-(\alpha(T)+T \beta(T)) \operatorname{grad} T) .
\end{aligned}
$$

At the interface, the following relation [50] holds:

$$
\operatorname{div} \mathbf{i}+I_{q n 1}-I_{q n 2}=-\frac{\partial \sigma}{\partial t}
$$

Here $\varepsilon$ is the permittivity, $c^{2}=1 /\left(\varepsilon_{0} \cdot \mu_{0}\right), \mu$ is the magnetic permeability, $\varepsilon_{0}$ is the electric constant, $\mu_{0}$ is the magnetic constant, $c_{p} \rho$ is the product of the specific heat of the medium by its density, and $\beta(T)=\partial \alpha(T) / \partial T$.

In deriving (60), use has been made of the condition of local electroneutrality of the substance. We give a differential formulation of the problem and the method of its solution in greater detail.

Let us investigate, on the segment $[0 \leq x<l]=[0 \leq x<$ $\xi] \cup[\xi \leq x \leq l]$, the contact of homogeneous media 1 and 2 with different electrophysical properties. The quantities $\varepsilon, \lambda, \mu, E$, and $\varphi$ have discontinuities of the first kind at the point of the interface $x=\xi$. We will consider the case of plane contact where the influence of surface currents can be disregarded and the thickness of the electric double-layer is much smaller than the characteristic dimension of the object. We set $E=-\operatorname{grad} \varphi$. Equations (59) and (60) for the onedimensional problem will take the form

$$
\begin{gathered}
\frac{\varepsilon}{c^{2}} \frac{\partial^{2} E}{\partial t^{2}}+\mu_{0} \frac{\partial}{\partial t}\left(-\lambda\left(\alpha(T) \frac{\partial T}{\partial x}-E\right)\right)=\frac{1}{\mu} \frac{\partial^{2} E}{\partial x^{2}} \\
c_{p} \rho \frac{\partial T}{\partial t}=\frac{\partial}{\partial x}\left(k(T) \frac{\partial T}{\partial x}\right) \\
-\lambda\left(\alpha(T) \frac{\partial T}{\partial x}-E\right)\left(E-(\alpha(T)+T \beta(T)) \frac{\partial T}{\partial x}\right) .
\end{gathered}
$$


Conditions (39) and (61) will be written, respectively, in the form

$$
\begin{array}{r}
\left.\varepsilon_{1} \varepsilon_{0} E_{1}\right|_{x=\xi-0}-\left.\varepsilon_{2} \varepsilon_{0} E_{2}\right|_{x=\xi+0}=\left.\sigma\right|_{x=\xi} \\
\left.\lambda_{1}\left(E_{1}-\alpha_{1}(T) \frac{\partial T}{\partial x}\right)\right|_{x=\xi-0}-\left.\lambda_{2}\left(E_{2}-\alpha_{2}(T) \frac{\partial T}{\partial x}\right)\right|_{x=\xi+0} \\
=-\left.\frac{\partial \sigma}{\partial t}\right|_{x=\xi}
\end{array}
$$

By differentiating (63) with respect to time and taking into account (64), at the phase boundary we obtain the condition of equality of the total currents:

$$
\left.\left[\lambda\left(E-\alpha(T) \frac{\partial T}{\partial x}\right)+\varepsilon \varepsilon_{0} \frac{\partial E}{\partial t}\right]\right|_{x=\xi}=0 .
$$

Here and in what follows, for the arbitrary function $f$ we adopt the notation

$$
\left.[f]\right|_{x=\xi}=\left.f\right|_{x=\xi+0}-\left.f\right|_{x=\xi-0} .
$$

Equality (63) is a corollary of the relation $\lim _{x \rightarrow \varepsilon \pm 0} \operatorname{div} \mathbf{D}=\rho$ [50]. Taking into consideration the finiteness of the value of the space electric charge and the continuity of it, in the onedimensional case we obtain

$$
\left.\left[\varepsilon \varepsilon_{0} \frac{\partial E}{\partial x}\right]\right|_{x=\xi}=0
$$

Relations (65) and (67) reflect the laws of conservation and continuity of electric charge $[45,50]$. At the contact point, we also have the equality of the temperatures and the energy fluxes [45]:

$$
\begin{gathered}
{\left.[T]\right|_{x=\xi}=0} \\
{\left.\left[k(T) \frac{\partial T}{\partial x}+\lambda\left(\alpha(T) \frac{\partial T}{\partial x}-E\right)(\alpha(T) T+\varphi)\right]\right|_{x=\xi}=0}
\end{gathered}
$$

Thus, in the presence of the interaction of the electric and thermal fields in a layered medium, the equality of the charge fluxes (65), the equality of the charges (67) (when the conditions of quasineutrality of the contacting media beyond the electric double-layer are satisfied), the equality of the temperatures (68), and the equality of the energy fluxes must be fulfilled at the interface of the media. In the relations for the charge and energy fluxes, we take into account crossthermoelectrical phenomena.

The process of charge of the electric field is considered on the finite time interval $\left[0 \leq t \leq t_{0}\right]$. The initial conditions have the form

$$
T(x, 0)=T_{0}(x), \quad E(x, 0)=f_{1}(x), \quad \frac{\partial E(x, 0)}{\partial t}=f_{2}(x) .
$$

We give the boundary conditions, for example, for the value $x=0$. We set the value of the total-current density to be known

$$
\left.\left(\lambda\left(\alpha(T) \frac{\partial T}{\partial x}-E\right)-\varepsilon \varepsilon_{0} \frac{\partial E}{\partial t}\right)\right|_{x=0}=j_{1}(t)
$$

and take into account the heat exchange at the boundary using the Newton relation

$$
\begin{array}{r}
\left.\left(k(T) \frac{\partial T}{\partial x}+\lambda\left(\alpha(T) \frac{\partial T}{\partial x}-E\right) \alpha(T) T+\varphi\right)\right|_{x=0} \\
=\left.\gamma_{1}\left(T-T^{*}\right)\right|_{x=0}
\end{array}
$$

where $\gamma_{1}$ is the coefficient of heat exchange and $T^{*}$ is the ambient temperature.

We consider the analogous relations at the right-hand boundary.

3.3.3. Interaction of Electric and Thermal Fields with Allowance for Mass Transfer. Let us consider the nonstationary model of heat and mass transfer for electrochemical systems with the example of electrolysis. For this problem, an electric double-layer occurs at the site of contact of the electrolyte with the metallic anode and cathode. Let us set the density of the space charge beyond the electric double-layer of the metal-electrolyte contact to be zero at the initial instant and hence, according to [51], remain constant in the future while the voltage drop in the electrodes and leads is small. The influence of the electrodes on the temperature field of the electrolyte will be taken into account in terms of the coefficient of heat exchange.

Two approaches to modeling of diffusion-electrical phenomena have currently been developed, each of which has certain disadvantages and advantages. The first approach $[37,38,42]$ is characterized by consideration of the flows of ions of the corresponding sort in a completely or partially dissociated electrolyte. The equations derived contain many parameters, the reliable procedure of determination of which is absent in the majority of cases. Furthermore, the proposed theory fails to provide for taking into account the interaction of the cations and the anions of the dissolved substance with each other and with the molecules of the solvent. It must also be borne in mind that, apart from the dissociation reaction, we have the association of simple molecules which in turn are dissociated into complex and simple ions. It is difficult to determine the diffusion coefficients of complex ions; it is also difficult to take into account the force interaction of complex cations and anions with each other and with the external electric field. The difficulties in question become more serious when a multicomponent electrolyte is described. We note that such difficulties also arise in modeling, for example, certain problems of plasma physics.

The second approach [52-56] fails to provide for the introduction and determination of the coefficients of molecular diffusion of cations and anions and the degree of dissociation of the electrolyte. It is based on the interaction of the mass and charge fluxes. The ionic flows are not determined. The model is formulated without explicit separation of the charge carriers and the charge of an electric double layer. 
The system of equations describing the electrodynamic processes in the electrolyte is as follows:

$$
\begin{gathered}
\frac{\varepsilon}{c^{2}} \frac{\partial^{2} \mathbf{E}}{\partial t^{2}}+\mu_{0} \frac{\partial \mathbf{I}_{q}}{\partial t}=\frac{1}{\mu} \nabla^{2} \mathbf{E}, \\
\frac{\partial n}{\partial t}=-\operatorname{div}\left(\mathbf{I}_{m}\right) \\
c_{0} \rho_{0} \frac{\partial T}{\partial t}=-\operatorname{div}\left(\mathbf{I}_{T}\right) .
\end{gathered}
$$

Here $\mathbf{I}_{q}, \mathbf{I}_{m}$, and $\mathbf{I}_{T}$ are the densities of the conduction current and of the mass and heat fluxes which (in the case of the nonequilibrium state of the medium) have, respectively, the form (57) [45], where $D_{M}$ is the molecular diffusion coefficient, $D_{A}^{*}$ is the ambipolar diffusion coefficient, $D_{T}$ is the thermal diffusion coefficient, $D_{T}^{*}$ is the coefficient taking into account the transfer of heat due to the motion of the impurity, and $n$ is the concentration of the impurity.

We give the initial

$$
\begin{array}{ll}
E(x, 0)=E_{0}(x), & \frac{\partial E(x, 0)}{\partial t}=\widetilde{E}(x), \\
T(x, 0)=T_{0}(x), & n(x, 0)=n_{0}(x),
\end{array}
$$

and boundary conditions (anode-electrolyte, $x=0$ ):

$$
\begin{gathered}
\mathbf{I}_{q}=\lambda\left(\lambda_{A}^{*} \frac{\partial n}{\partial x}-E\right)+\lambda \alpha(T) \frac{\partial T}{\partial x}-\varepsilon \varepsilon_{0} \frac{\partial E}{\partial t}, \\
\gamma_{1}\left(T-T^{*}\right)=k \frac{\partial T}{\partial x}-I_{q}(\Pi+\varphi)+D_{T}^{*} \frac{\partial n}{\partial x}, \\
k_{a} I_{q}(t)=D_{M} \frac{\partial n}{\partial x}-D_{A}^{*} \lambda E+D_{T} \frac{\partial T}{\partial x},
\end{gathered}
$$

in modeling the one-dimensional problem in the region $G=$ $[0 \leq x \leq l] \times[0 \leq t \leq T]$. Here $k_{a}$ is the electrochemical equivalent of a substance which deposits at the anode. The analogous conditions hold for $x=l$ as well.

The constructed system of differential equations makes it possible to model the processes of transfer in the electrochemical system with allowance for the influence of an electric double-layer; this influence directly determines the thermoelectrical and ambipolar electrodiffusion phenomena at the boundary of the metal-electrolyte contact.

\subsubsection{Numerical Modeling of Electrochemical Systems. Heating} of an Electrochemical Cell in the Case of Constant-Current and Pulse Electrolysis. In electrochemical systems, the electrodes are metals as a rule. The electrical conductivity of metals is hundreds of times higher than the specific electrical conductivity of electrolytes; therefore, one can disregard the voltage drop in electrodes and leads. In highly conductive metals, one can also disregard the displacement currents and the coefficients of ambipolar diffusion and thermal diffusion and restrict oneself to consideration of the system of equations (73)-(75) just in the electrolyte itself.

Let us consider the process of heating of an electrochemical medium in the case of passage of an electric current with density $I_{q}(t)$ through a solution of copper sulfate $\mathrm{CuSO}_{4} \cdot 5 \mathrm{H}_{2} \mathrm{O}$. We use copper $(99.78 \%)$ as the anode.
The copper from the solution is deposited at the cathode. We set the current efficiencies for the copper equal to $100 \%$ and the electrochemical equivalent $k_{e}$ equal to $0.6588 \cdot 10^{-6} \mathrm{~kg} / \mathrm{C}$. The dependence of the specific electrical conductivity $\lambda(n)$ of the copper-plating electrolyte on the concentration of the copper sulfate in water is given in [57]; the dependence of $\lambda$ and $k$ on the temperature will be considered to be insignificant. In the calculations for $\mathrm{CuSO}_{4}$, we set $D_{M}=$ $5 \cdot 10^{-10} \mathrm{~m}^{2} / \mathrm{sec}, D_{A}^{*}=10^{-11} \lambda, \lambda_{A}^{*}=10^{-4} \lambda, \varepsilon=70$, and $\mu=1$ and disregard the effects of thermal diffusion. The distance between the cathode and the anode is $L=0.05 \mathrm{~m}$. The heat capacity, the density, and the thermal-conductivity coefficient of the electrolyte are taken to be $4.2 \cdot 10^{3} \mathrm{~J} /(\mathrm{kg} \cdot \mathrm{K})$, $10^{3} \mathrm{~kg} / \mathrm{m}^{3}$, and $0.6 \mathrm{~V} /(\mathrm{m} \cdot \mathrm{K})$ respectively. We note that the regimes of pulse action have been considered in [58] as applied to the processes of electrodeposition of alloys. This investigation has been carried out on the basis of a separate description of the transport of ions and the employment of the Kirchhoff law for quasistationary currents in a cell. With such an approach, the displacement currents are taken into account indirectly through introduction of the capacitive current of the electrode and determination of the experimental dependence of the polarization capacitance of the electrode on the character of the pulse action. In [58], it has been noted that neglect of the capacitive current in pulse electrolysis involves significant errors. The employment of system (73)-(75) to model the process of copper plating makes it possible to consider the problems without introducing the concepts of inductance and capacitance. The obtained nonlinear system of equations is solved by the finite-difference method analogously to [54].

We have modeled numerically the process of copper plating for a constant current and a pulse current (Figure 16). The density of the constant current was equal to $300 \mathrm{~A} / \mathrm{m}^{2}$. The maximum density of the pulse current was also $300 \mathrm{~A} / \mathrm{m}^{2}$, while the period of traversal of the current and the break were equal to $0.01 \mathrm{sec}$.

Figure 16 gives the results of modeling the distributions of the concentration of $\mathrm{CuSo}_{4}$ and the temperature for different regimes of electrolysis. The measurements were carried out within $60 \mathrm{sec}$ after switching on the current. It is seen from the figure that the concentration gradients near the cathode surface $(x=1)$ are different and depend on the regime of copper plating. In the case of nonstationary action they are substantially smaller than in the case of electrolysis in the regime of constant current. As has been shown in [58], this tendency also holds in the case where one and the same total charge passes through the electrochemical cell.

The analysis of the temperature field (Figure 16(b)) shows that the temperatures of the electrolyte in the vicinity of the anode and the cathode differ significantly. Nonsymmetric heating of the electrochemical cell attributed to the am-bipolar diffusion and electrical ambipolar conductivity occurs. The modeling results are in agreement with the data of [59].

With the aim of measuring the temperature in the surface layer of the electrolyte solution near the cathode and comparing the results to the calculated data, we developed and manufactured an experimental setup. Copper anode and 


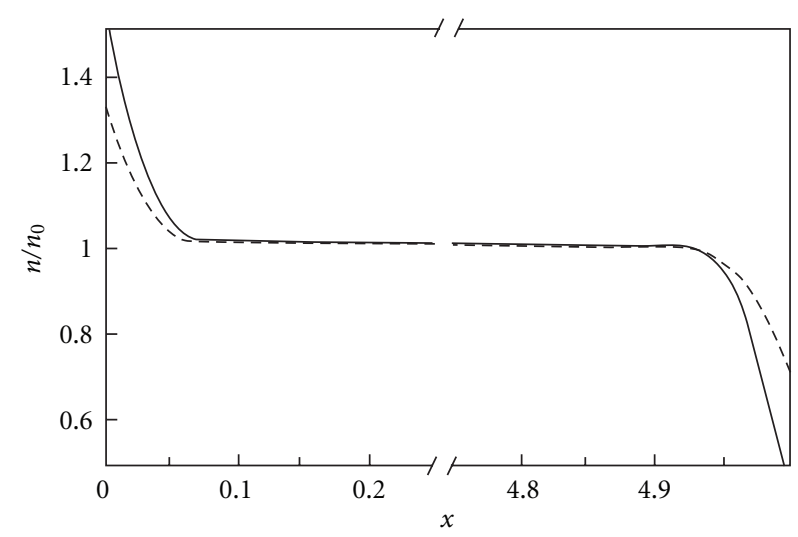

(a) Distribution of the normalized concentration of $\mathrm{CuSO}_{4}$

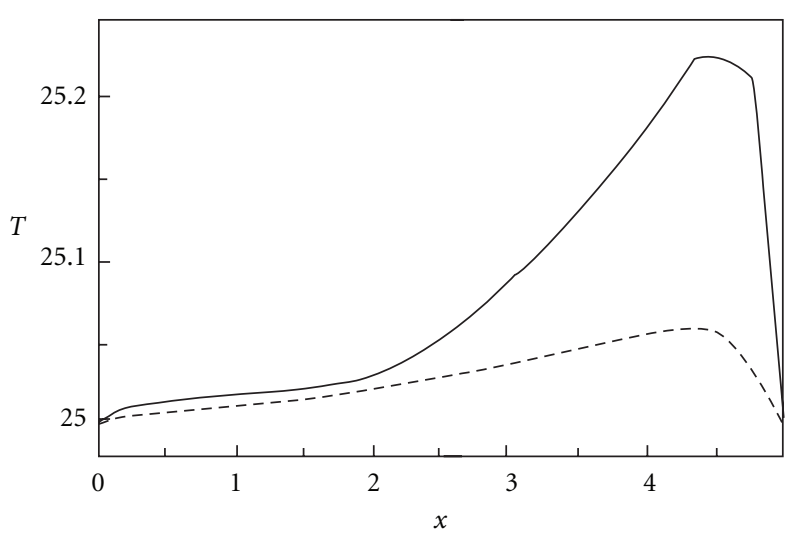

(b) Distribution of the temperature

FIGURE 16: Distribution of the normalized concentration of $\mathrm{CuSO}_{4}$ and the temperature in an electrochemical cell within 60 sec after switching on the current. The solid curve, at constant current; the dashed curve, at pulse current. $T$, ${ }^{\circ} \mathrm{C} ; x, \mathrm{~mm}$.

cathode were placed in an electroplating bath with a sulfuricacid copper-plating electrolyte. In the $1 \mathrm{~mm}$ thick cathode, a hole was made into which we placed a temperature-sensitive element. As the latter we employed a thermal resistor. The current strength in the bath was prescribed by a variable resistor.

Figure 17 gives the results of numerical modeling and the experimental data on heating of the cathode region in relation to the regime of current. It is seen that the increase in the density of the current (both constant and pulse) causes the temperature near the cathode surface to increase. In the anode, no increase in the temperature was observed either in the calculations or in the experiment, which is in agreement with the data of [59]. The surface temperature obtained in solving (73)-(75) in the regime of constant current is $10-15 \%$ higher than the temperature recorded in the experiments.

A more complicated situation occurs when we compare the experimental data and the results of numerical modeling in the regime of pulse electrolysis. The results of the experiments demonstrate that in the regime of pulse current the temperature of the electrolyte in the cathode region increases more than in the regime of constant current. At the same time, the numerical calculations of the pulse electrolysis show that the heating of the cathode region here is smaller than for the regime of constant current. The difference is, apparently, attributed to concentration convection [60], whose influence is substantial for high densities of the current for pulse electrolysis.

In a longer term, one must take into account natural and concentration convection and heat exchange with the ambient medium. Nonetheless, despite the errors in determination of the coefficients and the assumptions made in the model, the calculation results are in qualitative agreement with the experimental data, which makes it possible, in certain cases, to use the proposed approach for modeling of nonstationary processes.

Results were published in part $[52,53,55,56,61]$.

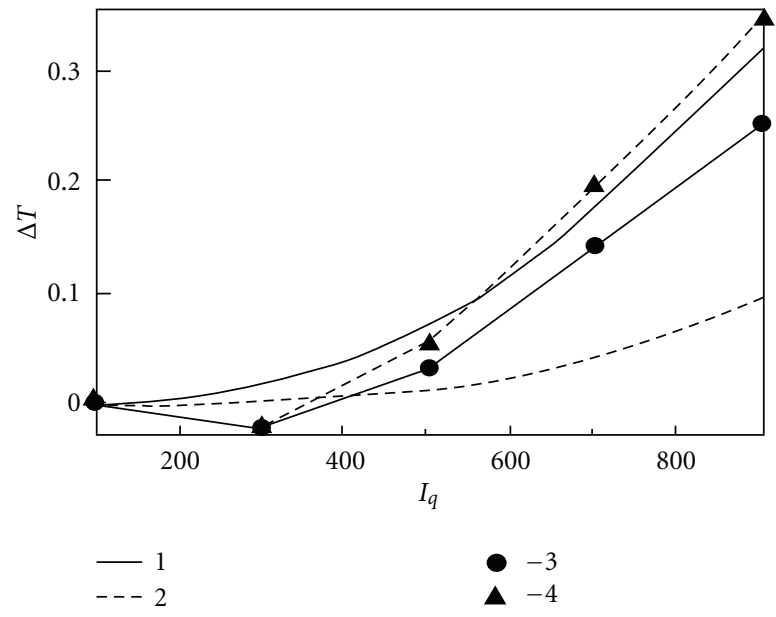

FIGURe 17: Heating of the electrolyte in the cathode region as a function of the value and kind of polarizing current: (1) constant current; (2) pulse current; $(3,4)$ corresponding experimental data. $\Delta T,{ }^{\circ} \mathrm{C} ; I_{q}, \mathrm{~A} / \mathrm{m}^{2}$.

\section{Interaction of Nonstationary Electric and Thermal Fields with Allowance for Relaxation Processes}

We investigate electric and thermal fields created by macroscopic charges and currents in continuous media. Of practical interest is modeling of local heat releases in media on exposure to a high-frequency electromagnetic field. We should take into account the influence of the energy absorption on the propagation of an electromagnetic wave, since the transfer processes are interrelated.

In an oscillatory circuit with continuously distributed parameters, the energy dissipation is linked [62] to the dielectric loss due to the dependence of the relative permittivity $\varepsilon(\omega)$ on frequency. In the general case $\varepsilon$ is also complex, 
and the relationship between the electric displacement and electric field vectors has the form $D=\varepsilon(\omega) E$, where $\varepsilon(\omega)=$ $\varepsilon^{\prime}(\omega)-i \mathcal{E}^{\prime \prime}(\omega)$; here, $\varepsilon^{\prime}$ and $\varepsilon^{\prime \prime}$ are determined experimentally. As of now, the problems of dielectric heating of a continuous medium are reduced in many cases to consideration of an equivalent circuit based on lumped parameters, such as capacitance, inductance, loss angle, and relative-dielectricloss factor $[63,64]$, that are established experimentally.

With this approach, there arise substantial difficulties in determining the temperature field of equivalent circuits. Also, we have polarization and the occurrence of an electric double-layer of a prescribed electric moment in contact of media with different properties. Equivalent circuits in lamellar media additionally involve empirical lumped parameters: surface capacitance and surface resistance [65]. The total current can always be separated into a dissipative or conduction current which is in phase with the applied voltage and a displacement current shifted in time relative to the voltage. The exact physical meaning of these components of the current is largely dependent on selection of an equivalent electric circuit. A unique equivalent circuitseries or parallel connection of the capacitor, the resistor, and the inductor-does not exist; it is determined by a more or less adequate agreement with experimental data.

In the case of electrolytic capacitors, the role of one plate is played by the electric double-layer with a specific resistance much higher than the resistance of metallic plates. Therefore, decrease in the capacitance with frequency is observed, for such capacitors, even in the acoustic-frequency range [65]. Circuits equivalent to an electrolytic capacitor are very bulky: up to $12 R, L$, and $C$ elements can be counted in them; therefore, it is difficult to obtain a true value of, for example, the electrolyte capacitance. In [65] experimental methods of measurement of the dielectric properties of electrolyte solutions at different frequencies are given and $\varepsilon^{\prime}$ and $\varepsilon^{\prime \prime}$ are determined. The frequency dependence of dispersion and absorption are essentially different consequences of one phenomenon: "dielectric-polarization inertia" [65]. In actual fact, the dependence $\varepsilon(\omega)$ is attributable to the presence of the resistance of the electric double-layer and to the electrochemical cell in the electrolytic capacitor being a system with continuously distributed parameters, in which the signal velocity is a finite quantity.

Actually, $\varepsilon^{\prime}$ and $\varepsilon^{\prime \prime}$ are certain integral characteristics of a material at a prescribed constant temperature, which are determined by the geometry of the sample and the properties of the electric double-layer. It is common knowledge that in the case of a field arbitrarily dependent on time any reliable calculation of the absorbed energy in terms of $\varepsilon(\omega)$ turns out to be impossible [45]. This can only be done for a specific dependence of the field $E$ on time. For a quasimonochromatic field, we have [45]

$$
\begin{aligned}
\mathbf{E}(t) & =\frac{1}{2}\left[\mathbf{E}_{0}(t) e^{-i \omega t}+\mathbf{E}_{0}^{*}(t) e^{i \omega t}\right], \\
\mathbf{H}(t) & =\frac{1}{2}\left[\mathbf{H}_{0}(t) e^{-i \omega t}+\mathbf{H}_{0}^{*}(t) e^{i \omega t}\right] .
\end{aligned}
$$

The values of $\mathbf{E}_{0}(t)$ and $\mathbf{H}_{0}(t)$, according to [66], must very slowly vary over the period $T=2 \pi / \omega$. Then, for absorbed energy, on averaging over the frequency $\omega$, we obtain the expression [66]:

$$
\begin{aligned}
\frac{\partial \mathbf{D}(t)}{\partial t} E(t)= & \frac{1}{4} \frac{d\left(\omega \varepsilon^{\prime}(\omega)\right)}{d(\omega)} \frac{\partial}{\partial t}\left[\mathbf{E}_{0}(t) \mathbf{E}_{0}^{*}(t)\right] \\
& +\frac{\omega \varepsilon^{\prime \prime}}{2} \mathbf{E}_{0}(t) \mathbf{E}_{0}^{*}(t) \\
& +i \frac{d\left(\omega \varepsilon^{\prime \prime}(\omega)\right)}{4 d \omega}\left(\frac{\partial \mathbf{E}_{0}(t)}{\partial t} \mathbf{E}_{0}^{*}(t)-\frac{\mathbf{E}_{0}^{*}(t)}{\partial t} \mathbf{E}_{0}(t)\right)
\end{aligned}
$$

where the derivatives with respect to frequency are taken at the carrier frequency $\omega$. We note that for an arbitrary function $\mathbf{E}(t)$, it is difficult to represent it in the form

$$
E(t)=a(t) \cos \varphi(t)
$$

since we cannot unambiguously indicate the amplitude $a(t)$ and the phase $\varphi(t)$. The manner in which $E(t)$ is decomposed into factors $a$ and $\cos \varphi$ is not clear. Even greater difficulties appear in the case of going to the complex representation $W(t)=U(t)+i V(t)$ when the real oscillation $E(t)$ is supplemented with the imaginary part $V(t)$. The arising problems have been considered in [67] in detail. In the indicated work, it has been emphasized that certain methods using a complex representation and claiming higher-thanaverage accuracy become trivial without an unambiguous determination of the amplitude, phase, and frequency.

Summing up the aforesaid, we can state that calculation of the dielectric loss is mainly empirical in character. Construction of the equivalent circuit and allowance for the influence of the electric double-layer and for the dependence of electrophysical properties on the field's frequency are only true of the conditions under which they have been modeled; therefore, these are fundamental difficulties in modeling the propagation and absorption of electromagnetic energy.

As we believe, the release of heat in media on exposure to nonstationary electric fields can be calculated on the basis of allowance for the interaction of electromagnetic and thermal fields as a system with continuously distributed parameters from the field equation and the energy equation which take account of the distinctive features of the boundary between adjacent media. When the electric field interacting with a material medium is considered we use Maxwell equations (6)-(7). We assume that space charges are absent from the continuous medium at the initial instant of time and they do not appear throughout the process. The energy equation will be represented in the form

$$
\rho C_{p} \frac{d T}{d t}=\operatorname{div}[k(T) \operatorname{grad}(T)]+Q,
$$

where $Q$ is the dissipation of electromagnetic energy.

According to [68], the electromagnetic energy converted to heat is determined by the expression

$$
Q=\rho\left[\mathbf{E} \frac{d}{d t}\left(\frac{\mathbf{D}}{\rho}\right)+H \frac{d}{d t}\left(\frac{\mathbf{B}}{\rho}\right)\right]+\mathbf{J}_{q} E .
$$

In deriving this formula, we used the nonrelativistic approximation of Minkowski's theory. If $\varepsilon, \mu$, and $\rho=$ const, there is 
no heat release; therefore, the intrinsic dielectric loss is linked to the introduction of $\varepsilon^{\prime}(\omega)$ and $\varepsilon^{\prime \prime}(\omega)$. The quantity $Q$ is affected by the change in the density of the substance $\rho(T)$.

A characteristic feature of high frequencies is the lag of the polarization field behind the charge in the electric field in time. Therefore, the electric-polarization vector is expediently determined by solution of the equation $\mathbf{P}(t+$ $\left.\tau_{e}\right)=(\varepsilon-1) \varepsilon_{0} \mathbf{E}(t)$ with allowance for the time of electric relaxation of dipoles $\tau_{e}$. Restricting ourselves to the first term of the expansion $\mathbf{P}\left(t+\tau_{e}\right)$ in a Taylor series, from this equation, we obtain

$$
\mathbf{P}(t)+\tau_{e} \frac{d \mathbf{P}(t)}{d t}=(\varepsilon-1) \varepsilon_{0} \mathbf{E}(t)
$$

The solution (81), on condition that $\mathbf{P}=0$ at the initial instant of time, will take the form

$$
\mathbf{P}=\frac{(\varepsilon-1) \varepsilon_{0}}{\tau_{e}} \int_{t_{0}}^{t} \mathbf{E}(\tau) e^{-(t-\tau) / \tau_{e}} d \tau
$$

It is noteworthy that (81) is based on the classical Debay model. According to this model, particles of a substance possess a constant electric dipole moment. The indicated polarization mechanism involves partial arrangement of dipoles along the electric field, which is opposed by the process of disorientation of dipoles because of thermal collisions. The restoring "force", in accordance with (81), does not lead to oscillations of electric polarization. It acts as if constant electric dipoles possessed strong damping.

Molecules of many liquids and solids possess the Debay relaxation polarizability. Initially polarization aggregates of Debay oscillators turn back to the equilibrium state $\mathbf{P}(t)=$ $\mathbf{P}(0) \exp (-t / \tau e)$.

A dielectric is characterized, as a rule, by a large set of relaxation times with a characteristic distribution function, since the potential barrier limiting the motion of weakly coupled ions may have different values [63]; therefore, the mean relaxation time of the ensemble of interacting dipoles should be meant by $\tau_{e}$ in $(81)$.

To eliminate the influence of initial conditions and transient processes we set $t_{0}=-\infty, \mathbf{E}(\infty)=0, \mathbf{H}(\infty)=0$, as it is usually done. If the boundary regime acts for a fairly long time, the influence of initial data becomes weaker with time owing to the friction inherent in every real physical system. Thus, we naturally arrive at the problem without the initial conditions:

$$
\mathbf{P}=\frac{(\varepsilon-1) \varepsilon_{0}}{\tau_{e}} \int_{-\infty}^{t} \mathbf{E}(\tau) e^{-(t-\tau) / \tau_{e}} d \tau
$$

Let us consider the case of the harmonic field $\mathbf{E}=\mathbf{E}_{0} \sin \omega t$; then, using (83) we have, for the electric induction vector

$$
\begin{aligned}
\mathbf{D}=\varepsilon_{0} \mathbf{E}+\mathbf{P}= & \frac{(\varepsilon-1) \varepsilon_{0}}{\tau_{e}} \int_{-\infty}^{t} \mathbf{E}(\tau) e^{-(t-\tau) / \tau_{e}} d \tau+\varepsilon_{0} \mathbf{E}_{0} \sin \omega t \\
= & \frac{\mathbf{E}_{0}(\varepsilon-1) \varepsilon_{0}}{1+\omega^{2} \tau_{e}^{2}}\left(\sin \omega t-\omega \tau_{e} \cos \omega t\right) \\
& +\varepsilon_{0} \mathbf{E}_{0} \sin \omega t
\end{aligned}
$$

The electric induction vector is essentially the sum of two absolutely different physical quantities: the field strength and the polarization of a unit volume of the medium.

If the change in the density of the substance is small, we obtain, from formula (80), for the local instantaneous heat release

$$
\mathbf{Q}=\mathbf{E} \frac{d \mathbf{D}}{d t}=\frac{E_{0}^{2}(\varepsilon-1) \varepsilon_{0}}{1+\omega^{2} \tau_{e}^{2}}\left(\omega \sin \omega t \cos \omega t+\omega^{2} \tau_{e} \sin ^{2} \omega t\right)
$$

when we write the mean value of $Q$ over the total period $T$ :

$$
\mathbf{Q}=\frac{1}{2} \frac{\mathbf{E}_{0}^{2}(\varepsilon-1) \varepsilon_{0}}{1+\omega^{2} \tau_{e}^{2}} \omega^{2} \tau_{e}+\frac{\lambda \mathbf{E}^{2}}{2}
$$

For high frequencies $(\omega \rightarrow \infty)$, heat release ceases to be dependent on frequency, which is consistent with formula (86) and experiment [63].

When the relaxation equation for the electric field is used we must also take account of the delay of the magnetic field, when the magnetic polarization lags behind the change in the strength of the external magnetic field:

$$
\mathbf{I}(t)+\tau_{i} \frac{d \mathbf{I}(t)}{d t}=\mu \mu_{0} \mathbf{H}(t)
$$

Formula (86) is well known in the literature; it has been obtained by us without introducing complex parameters. In the case of "strong" heating of a material where the electrophysical properties of the material are dependent on temperature expression (81) will have a more complicated form and the expression for $Q$ can only be computed by numerical methods. Furthermore, in the presence of strong field discontinuities, we cannot in principle obtain the expression for $Q$ because of the absence of closing relations for the induced surface charge and the surface current on the boundaries of adjacent media; therefore, the issue of energy relations in macroscopic electrodynamics is difficult, particularly, with allowance for absorption.

Energy relations in a dispersive medium have repeatedly been considered; nonetheless, in the presence of absorption, the issue seems not clearly understood (or at least not sufficiently known), particularly in the determination of the expression of released heat on the boundaries of adjacent media.

Indeed, it is known from the thermodynamics of dielectrics that the differential of the free energy $F$ has the form

$$
d F=-S d T-P d V+E d D
$$

If the relative permittivity and the temperature and volume of the dielectric are constant quantities, from (88) we have

$$
F(T, D)=F_{0}+\varepsilon \varepsilon_{0} \frac{E^{2}}{2},
$$

where $F_{0}$ is the free energy of the dielectrics in the absence of the field.

The change of the internal energy of the dielectrics during its polarization at constant temperature and volume can 
be found from the Gibbs-Helmholtz equation. Disregarding $F_{0}$ which is independent of the field strength, we can obtain

$$
U(T, D)=F(T, D)-T\left(\frac{d F}{d T}\right)_{D} .
$$

If the relative dielectric constant is dependent on temperature $(\varepsilon(T))$, we obtain

$$
U(T, D)=\frac{\varepsilon_{0} E^{2}}{2}+\left(\varepsilon+T\left(\frac{d F}{d T}\right)_{D}\right) .
$$

Expression (91) determines the change in the internal energy of the dielectric in its isothermal polarization but with allowance for the energy transfer to a thermostat, if the polarization causes the dielectric temperature to change. In the works on microwave heating, that we know, (91) is not used.

A characteristic feature of high frequencies is that the polarization field lags behind the change in the external field in time; therefore, the polarization vector is expediently determined by solution of the equation:

$$
\mathbf{P}\left(t+\tau_{e}\right)=\left(\varepsilon-1+T\left(\frac{d \varepsilon}{d T}\right)_{D}\right) \varepsilon_{0} \mathbf{E}(t) .
$$

With allowance for the relaxation time, that is, restricting ourselves to the first term of the expansion $\mathbf{P}\left(t+\tau_{e}\right)$ in a Taylor series, we obtain

$$
\mathbf{P}(t)+\tau_{e} \frac{d \mathbf{P}(t)}{d T}=\left(\varepsilon-1+T\left(\frac{d \varepsilon}{d T}\right)_{D}\right) \varepsilon_{0} \mathbf{E}(t) .
$$

In the existing works on microwave heating with the use of complex parameters, they disregard the dependence $\varepsilon^{\prime \prime}(T)$. In [69], consideration has been given to the incidence of a one-dimensional wave from a medium with arbitrary complex parameters on one or two boundaries of media whose parameters are also arbitrary. The amplitudes of waves reflected from and transmitted by each boundary have been found. The refection, transmission, and absorption coefficients have been obtained from the wave amplitudes. The well-known proposition that a traditional selection of determinations of the reflection, transmission, and absorption coefficients from energies (reflectivity, transmissivity, and absorptivity) in the case of complex parameters of media comes into conflict with the law of conservation of energy has been confirmed and exemplified. The necessity of allowing for $\varepsilon^{\prime \prime}(T)$ still further complicates the problem of computation of the dissipation of electromagnetic energy in propagation of waves through the boundaries of media with complex parameters.

The proposed method of computation of local heat release is free of the indicated drawbacks and makes it possible, for the first time, to construct a consistent model of propagation of nonmonochromatic waves in a heterogeneous medium with allowance for frequency dispersion without introducing complex parameters.

In closing, we note that a monochromatic wave is infinite in space and time, has infinitesimal energy absorption in a material medium, and transfers infinitesimal energy, which is the idealization of real processes. However with these stringent constraints, too, the problem of propagation of waves through the boundary is open and far from being resolved even when the complex parameters of the medium are introduced and used. In reality, the boundary between adjacent media is not infinitely thin and has finite dimensions of the electric double-layers; therefore, approaches based on through-counting schemes for a hyperbolic equation without explicit separation of the boundary between adjacent media are promising.

\section{Ponderomotive Forces in Heterogeneous Lamellar Media with Absorption}

It is common knowledge that the electric field strength $\mathbf{E}$ is equal to the ponderomotive mechanical force per unit charge acting at a given point of the field on a test charge (charged body). The issue of the force acting on induced surface charges on the boundaries remains to be solved, since there are no closing equations for $\sigma$ and the charge is not determined on the interface itself. According to [45], the tension tensor of ponderomotive forces for media with dispersion and absorption due to the dipole relaxation cannot be found in general form in a macroscopic manner at all. As we believe, solution of this problem lies in computing the induced surface charge on the boundaries and the resultants of all tensions applied externally to the surface without the necessity of reducing bulk ponderomotive forces to tensions. The reduction of bulk forces to tensions is not necessarily possible; therefore, this issue will be considered in greater detail.

5.1. Reduction of Bulk Ponderomotive Forces to Tensions. If $\mathbf{f}$ is the bulk density of ponderomotive forces, the resultant of all forces applied to the bodies within the volume $V$ is

$$
\mathbf{F}=\int_{V} \mathbf{f} d V .
$$

On the other hand, if bulk forces can be reduced to tensions at all, the set of tensions acting externally of a closed surface $S$ must be equal to the same quantity. We denote by $T_{n}$ a force acting externally per unit surface the external normal to which is directed along $n$; the components of this force along the coordinate axes are $T_{x n}, T_{y n}$ and $T_{z n}$. Then the resultant of all tensions applied externally to the surface will be

$$
\mathbf{F}=\oint_{\mathrm{s}} \mathbf{T}_{n} d S .
$$

Using the Ostrogradsky-Gauss theorem and equating (94) and (95), we obtain

$$
\begin{aligned}
& f_{x}=\frac{\partial T_{x x}}{\partial x}+\frac{\partial T_{x y}}{\partial y}+\frac{\partial T_{x z}}{\partial z} \\
& f_{y}=\frac{\partial T_{y x}}{\partial x}+\frac{\partial T_{y y}}{\partial y}+\frac{\partial T_{y z}}{\partial z} \\
& f_{z}=\frac{\partial T_{z x}}{\partial x}+\frac{\partial T_{z y}}{\partial y}+\frac{\partial T_{z z}}{\partial z} .
\end{aligned}
$$


For the bulk forces and tensions to be equivalent, it is necessary that not only the resultant of forces applied to an arbitrary volume remains constant but the moment of these forces be invariant as well [70]. This circumstance imposes an additional constraint on the tension-tensor components. The moment of bulk forces applied to an arbitrary volume $V$ is

$$
\mathbf{N}=\int\lfloor\mathbf{R} \mathbf{f}\rfloor d V
$$

where $\mathbf{R}$ is the distance from point $O$ for which the moment of forces is determined to the element $d V$. Consequently, the component $N_{x}$ must satisfy relation (97):

$$
\begin{aligned}
N_{x} & =\int_{V}\left(y f_{z}-z f_{y}\right) d V \\
& =\oint_{\mathrm{s}}\left(y T_{z n}-z T_{y n}\right) d S+\int_{V}\left(T_{y z}-T_{z y}\right) d V .
\end{aligned}
$$

The surface integral on the right is equal to the moment of tension forces $T_{n}$ applied to the surface $S$ of volume $V$. The moment of these tension forces will be equal to the moment of bulk forces only in the case where the last integral on the right is equal to zero. In view of the arbitrariness of the volume $V$, this will occur only when the integrand

$$
T_{y z}=T_{z y}
$$

is equal to zero.

By repeating the same considerations for $N_{y}$ and $N_{z}$, we obtain $T_{z x}=T_{x y}, T_{x y}=T_{y x}$. Consequently, for the bulk forces and surface ponderomotive forces to be equivalent, the tension tensor must be symmetric. In anisotropic media, the tension tensor is asymmetric; therefore, direct computation of the resultant of forces in terms of tensions from formula (95) is expedient. We note that Maxwell was the first to reduce the field's ponderomotive forces to tensions. Replacement of ponderomotive forces by an equivalent system of tensions makes it much easier to determine forces applied to the volume of the electric field.

The force applied to a charged layer, given the uncompensated surface charge, has the form

$$
F_{i j}^{S}=\sigma\left(E_{i j}^{1}-E_{i j}^{2}\right)
$$

If the layer is not charged, we have $\sigma=0$ and $F_{i j}^{S}=0$. The resultant force applied to the boundary of a cell is

$$
\mathbf{F}=\oint \mathbf{F}_{i j} d S
$$

The projections of $f_{x}, f_{y}$, and $f_{z}$ are determined according to (96), and in a variable electromagnetic field, we must take into account that $f_{x}, f_{y}$, and $f_{z}$ are expended in changing the density of the momentum flux of the substance and the electromagnetic field alike. If the forces acting only on the substance are meant by $\tilde{f}_{x}, \tilde{f}_{y}, \tilde{f}_{z}$, according to [70], we have

$$
\begin{aligned}
& \tilde{f}_{x}=f_{x}-(\varepsilon \mu-1) \frac{\partial}{\partial t}[\mathbf{E} \overrightarrow{\mathbf{H}}]_{x}, \\
& \tilde{f}_{y}=f_{y}-(\varepsilon \mu-1) \frac{\partial}{\partial t}[\mathbf{E} \overrightarrow{\mathbf{H}}]_{y}, \\
& \tilde{f}_{z}=f_{z}-(\varepsilon \mu-1) \frac{\partial}{\partial t}[\mathbf{E} \overrightarrow{\mathbf{H}}]_{z} .
\end{aligned}
$$

The expression $(\varepsilon \mu-1)(\partial / \partial t)[\mathbf{E} \overrightarrow{\mathbf{H}}]$ is known to be called the Abraham force [70].

If the medium is heterogeneous and the transmission of the electromagnetic wave involves its absorption and heating, we propose the following scheme of calculating ponderomotive forces:

(1) the domain under study is subdivided into $\mathrm{N}$ cells with constant properties;

(2) by the method presented in Para 1 and Para 2, we compute the surface charge $\sigma$ and the surface ponderomotive force for each cell.

In this approach, we disregard the influence of striction forces and tensions acting only on the force distribution over the body's volume but exerting no influence on the value of the resultant of all forces and on their moment [70]. Allowance for ponderomotive forces is important, for example, for biomechanics problems.

The history of the problem of ponderomotive forces developed into a paradoxical situation where the issues of certain basic propositions of classical electrodynamics have been discussed to date [66].

\section{Conclusion}

The consistent physicomathematical model of propagation of an electromagnetic wave in a heterogeneous medium has been constructed using the generalized wave equation and the Dirichlet theorem. Twelve conditions at the interfaces of adjacent media were obtained and justified without using a surface charge and surface current in explicit form. The conditions are fulfilled automatically in each section of the heterogeneous medium and are conjugate, which made it possible to use through-counting schemes for calculations. For the first time the effect of concentration of "mediumfrequency" waves with a length of the order of hundreds of meters at the fractures and wedges of domains of size $1-3 \mu \mathrm{m}$ has been established. Numerical calculations of the total electromagnetic energy on the wedges of domains were obtained. It is shown that the energy density in the region of wedges is maximum and in some cases may exert an influence on the motion, sinks, and the source of dislocations and vacancies and, in the final run, improve the near-surface layer of glass due to the "micromagnetoplastic" effect.

The results of these calculations are of special importance for medicine, in particular, when microwaves are used in the therapy of various diseases. For a small, on the 
average, permissible level of electromagnetic irradiation, the concentration of electromagnetic energy in internal angular structures of a human body (cells, membranes, neurons, interlacements of vessels, etc.) is possible.

For the first time, we have constructed a consistent physicomathematical model of interaction of nonstationary electric and thermal fields in a layered medium with allowance for mass transfer. The model is based on the methods of thermodynamics and on the equations of an electromagnetic field and is formulated without explicit separation of the charge carriers and the charge of an electric double-layer. We have obtained the relations for the electricfield strength and the temperature, which take into account the equality of the total currents and the energy fluxes, to describe the electric and thermal phenomena in layered media where the thickness of the electric double-layer is small compared to the dimensions of the object under study.

We have modeled numerically the heating of an electrochemical cell with allowance for the influence of the electric double-layer at the metal-electrolyte interface. The calculation results are in satisfactory agreement with experimental data.

\section{Notation}

$\overrightarrow{\mathrm{B}}$

D:

$D_{M}$ :

$D_{i}:$

$D_{A}$ :

$D_{A}^{*}$ :

E:

F:

F:

$F_{0}$ :

J:

$\overrightarrow{\mathrm{H}}$ :

$h$ :

$I_{q}, I_{q}:$

$\mathbf{i}_{\tau}$ :

i, j, k:

$\mathbf{j}_{\text {tot }}$ :

$L:$

$l:$ n:

$n$ :

$n_{A}$ :

$n_{i}:$

$n_{m}:$

$\mathrm{R}:$

$R:$

$P$ :

$q:$

$r:$

$S:$

T:

$t:$

$V:$

$v$ :

$U$ :

$W:$

$x, y, z$ :

$\beta$ :

$\alpha:$

$\varepsilon:$

$\mathcal{E}_{0}$ :

Electric displacement, $\mathrm{C} / \mathrm{m}^{2}$

Molecular diffusion

coefficient, $\mathrm{m}^{2} / \mathrm{sec}$

Diffusion coefficients of

anions and cations, $\mathrm{m}^{2} / \mathrm{sec}$

Ambipolar diffusion

coefficient, $\mathrm{A} /(\mathrm{m} \cdot \mathrm{kg})$

Ambipolar diffusion

coefficient, $\mathrm{kg} /(\mathrm{A} \cdot \mathrm{sec})$

Electric field strength, $\mathrm{V} / \mathrm{m}$

Free energy of the unit

volume of a dielectric, $\mathrm{J} / \mathrm{m}^{3}$

Faraday's constant,

96.985 C/eq.

Free energy of a dielectric in

the absence of a field, $\mathrm{J} / \mathrm{m}^{3}$

Current density, A/m ${ }^{2}$

Magnetic field strength, $\mathrm{A} / \mathrm{m}$

Thickness of the electric

double-layer, $\mathrm{m}$

Normal components of the

conduction current in

media 1 and $2, \mathrm{C} /\left(\mathrm{m}^{2} \cdot \mathrm{sec}\right)$

Surface current, $\mathrm{A} / \mathrm{m}$

Unit vectors of the

orthonormalized basis

Total current, A/m

Length, m

Average distance between

ferroparticles, $\mathrm{m}$

$\bar{\lambda}:$

$\mu:$

$\mu_{0}$ :
Unit vector normal to the

interface

Concentration, $\mathrm{kg} / \mathrm{m}^{3}$

Concentration of neutral

molecules, $\mathrm{kg} / \mathrm{m}^{3}$

Concentration of dissociated

molecules, $\mathrm{kg} / \mathrm{m}^{3}$

Molar concentration,

g. eq./liter

Universal gas constant

Resistance, $\Omega \cdot \mathrm{m}$

Pressure, $\mathrm{Pa}$

Mass flux, $\mathrm{kg} /\left(\mathrm{m}^{2} \cdot \mathrm{sec}\right)$

Radius of a particle, $\mu \mathrm{m}$

Interface between adjacent

media

Temperature, ${ }^{\circ} \mathrm{C}$

Time, sec

Volume, $\mathrm{m}^{3}$

Velocity of the slip of a

ferroparticle over the treated surface, $\mathrm{m} / \mathrm{sec}$

Voltage, $\mathrm{V}$

Magnetic field density, $\mathrm{J} / \mathrm{m}^{3}$

Cartesian coordinates;

Equilibrium constant

Degree of dissociation

Relative permittivity

Electric constant equal to

$8.58 \cdot 10^{-12} \mathrm{~F} / \mathrm{m}$

Electric potential, $\mathrm{V}$

Boltzmann constant;

Electrical conductivity, $\Omega \cdot \mathrm{m}$

Electrochemical equivalent

of copper, $\mathrm{kg} / \mathrm{C}$

Coefficient of specific

electrical ambipolar

conductivity, $\mathrm{V} \cdot \mathrm{m}^{3} / \mathrm{kg}$

Average value of electrical

conductivity, $\Omega \cdot \mathrm{m}$

Relative permeability

Magnetic constant equal to

$4 \pi \cdot 10^{-7} \mathrm{gf} / \mathrm{m}$

Chemical potential

Discontinuity point

Specific electric charge,

$\mathrm{C} / \mathrm{m}^{2}$

Conductance

Surface density of a charge,

$\mathrm{C} / \mathrm{m}^{2}$

Angular frequency, 1/sec

$\nabla: \frac{\partial}{\partial x}+\frac{\partial}{\partial y}+\frac{\partial}{\partial z}: \quad$ Symbolic operator

$\left.[f]\right|_{x=\xi}=\left.f_{1}\right|_{x=\xi+0}-\left.f_{2}\right|_{x=\xi-0}$ : Subscripts: 0, constant

component of a magnetic

field $H$

First medium

Second medium

Normal 


$\begin{array}{ll}q: & \text { Charge } \\ m: & \text { Impurity mass } \\ a: & \text { Anode } \\ A: & \text { Ambipolar } \\ M: & \text { Molecular } \\ e: & \text { Equivalent } \\ c: & \text { Concentration } \\ i: & \text { Number of the grid node } \\ j: & \text { Component } \\ \text { max: } & \text { Maximum } \\ n, \tau: & \text { Directions normal and } \\ & \text { tangential to the interface } \\ x: & \text { Normal component of a } \\ y, z: & \text { vector } \\ & \text { Tangential components of a } \\ \text { tot: } & \text { vector at the interface } \\ & \text { between adjacent media } \\ & \text { Total. }\end{array}$

\section{Acknowledgments}

The authors express their gratitude to Corresponding Member of the National Academy of Sciences of Belarus N.V. Pavlyukevich, Corresponding Member of the National Academy of Sciences of Belarus Professor V.I. Korzyuk for a useful discussion of the work. This work war carried out with financial support from the Belarusian Republic Foundation for Basic Research and from the Science Support Foundation of Poland "Kassa im. Myanowski" (2005).

\section{References}

[1] I. Monzon, T. Yonte, and L. Sanchez-Soto, "Characterizing the reflectance of periodic lasered media," Characterizing the reflectance of periodic lasered media, vol. 218, pp. 43-47, 2003.

[2] Y. Eremin and T. Wriedt, "Large dielectric non-spherical particle in an evanescent wave field near a plane surface," Optics Communications, vol. 214, no. 1-6, pp. 39-45, 2003.

[3] W. Hu and H. Guo, "Ultrashort pulsed Bessel beams and spatially induced group-velocity dispersio," Journal of the Optical Society of America A, vol. 19, no. 1, pp. 49-52, 2002.

[4] D. Danae, P. Bienstman, R. Bockstaele, and R. Baets, "Rigorous electromagnetic analysis of dipole emission in periodically corrugated layers: the grating-assisted resonant-cavity lightemitting diode," Journal of the Optical Society of America A, vol. 19, no. 5, pp. 871-880, 2002.

[5] J. I. Larruquert, "Reflectance enhancement with sub-quarterwave multilayers of highly absorbing materials," Journal of the Optical Society of America A, vol. 18, no. 6, pp. 1406-1414, 2001.

[6] B. M. Kolundzija, "Electromagnetic modeling of composite metallic and dielectric structures," IEEE Transactions on Microwave Theory and Techniques, vol. 47, no. 7, pp. 10211032, 1999.

[7] R. A. Ehlers and A. C. R. Metaxas, "3-D FE discontinuous sheet for microwave heating," IEEE Transactions on Microwave Theory and Techniques, vol. 51, no. 3, pp. 718-726, 2003.

[8] O. Bárta, J. Pištora, J. Vlček, F. Staněk, and T. Kreml, "Magneto-optics in bi-gyrotropic garnet waveguide," Opto-Electronics Review, vol. 9, no. 3, pp. 320-325, 2001.
[9] J. Broe and O. Keller, "Quantum-well enhancement of the Goos-Hänchen shift for p-polarized beams in a two-prism configuration," Journal of the Optical Society of America A, vol. 19, no. 6, pp. 1212-1222, 2002.

[10] O. Keller, "Optical response of a quantum-well sheet: internal electrodynamics," Journal of the Optical Society of America B, vol. 12, no. 6, pp. 997-1005, 1995.

[11] O. Keller, "Sheet-model description of the linear optical response of quantum wells," Journal of the Optical Society of America B, vol. 12, no. 6, pp. 987-997, 1995.

[12] O. Keller, "V: local fields in linear and nonlinear optics of mesoscopic system," Progress in Optics, vol. 37, pp. 257-343, 1997.

[13] G. A. Grinberg and V. A. Fok, "On the theory of Coastal Refraction of Electromagnetic Waves," in Investigations on Propagation of Radio Waves, B. A. Vvedenskii, Ed., M-L. AN SSSR, 1948.

[14] N. N. Grinchik and A. P. Dostanko, "Influence of Thermal and Diffusional Processes on the Propagation of Electromagnetic Waves in Layered Materials," Minsk: ITMO im. A. V. Luikova, NAN Belarusi, 2005.

[15] M. Born, Principles of Optics, Mir, Moscow, Russia, 1970.

[16] L. Kudryavtsev, Mathematical Analysis, Mir, Moscow, Russia, 1970.

[17] A. Frumkin, Electrode Processes, Nauka, Moscow, Russia, 1987.

[18] A. N. Tikhonov and A. A. Samarskii, Equations of Mathematical Physics, Nauka, Moscow, Russia, 1977.

[19] A. F. Kryachko et al., Theory of Scattering of Electromagnetic Waves in the Angular Structure, Nauka, Moscow, Russia, 2009.

[20] M. Leontovich, On the Approximate Boundary Conditions for the Electromagnetic Field on the Surface of Well Conducting Bodies, On the approximate boundary conditions for the electromagnetic field on the surface of well conducting bodies, Moscow, Russia, 1948.

[21] N. N. Grinchik, A. P. Dostanko, I. A. Gishkelyuk, and Y. N. Grinchik, "Electrodynamics of layered media with boundary conditions corresponding to the total-current continuum," Journal of Engineering Physics and Thermophysics, vol. 82, no. 4, pp. 810-819, 2009.

[22] Z. P. Shul'man and V. I. Kordonskii, Magnetorheological Effect, Nauka i Tekhnika, Minsk, Russia, 1982.

[23] M. Khomich, Magnetic-Abrasive Machining of the Manufactured Articles, BNTU, Minsk, Russia, 2006.

[24] M. N. Levin et al., "Activation of the surface of semiconductors by the effect of a pulsed magnetic field," Zh. Tekh. Fiz., vol. 73, no. 10, pp. 85-87, 2003.

[25] A. M. Orlov et al., "Magnetic- stimulated alteration of the mobility of dislocations in the plastically deformed silicon of n-type," Fiz. Tverd. Tela, vol. 43, no. 7, pp. 1207-1210, 2001.

[26] V. A. Makara et al., "On the influence of a constant magnetic field on the electroplastic effect in silicon crystals," Fiz. Tverd. Tela, no. 3, pp. 462-465, 2001.

[27] A. P. Rakomsin, Strengthening and Restoration of Items in an Electromagnetic Field, Paradoks, Minsk, Russia, 2000.

[28] Yu. I. Golovin et al., "Influence of weak magnetic fields on the dynamics of changes in the microhardness of silicon initiated by low-intensity beta-irradiation," Fiz. Tverd. Tela, vol. 49, no. 5.

[29] V. A. Makara et al., "Magnetic field-induced changes in the impurity composition and microhardness of the near-surface layers of silicon crystals," Fiz. Tekh. Poluprovadn, vol. 42, no. 9, pp. 1061-1064, 2008.

[30] A. M. Orlov et al., "Dynamics of the surface dislocation ensembles in silicon in the presence of mechanical and 
magnetic perturbation," Fiz. Tverd. Tela, vol. 45, no. 4, pp. 613-617, 2003.

[31] N. S. Akulov, Dislocations and Plasticity, Izd. AN BSSR, Minsk, Russia, 1961.

[32] N. S. Akulov, Ferromagnetism, ONTI, Leningrad, Russia, 1939.

[33] I. P. Bazarov, Thermodynamics: Textbook for Higher Educational Establishments, Vysshaya Shkola, Moscow, Russia, 1991.

[34] N. N. Grinchik et al., "Electrodynamic processes in a surface layer in magnetoabrasive polishing," Journal of Engeneering Physics and Thermodynamics, vol. 83, no. 3, pp. 638-649, 2010.

[35] A. Einstein, Elementary Theory of Brownian Motion, Collected Papers (3), 1966.

[36] V. E. Golant et al., Fundamental Principles of Plasma Physics, Moscow, Russia, 1977.

[37] Y. Kharkats, "Dependence of the limiting diffusion-migration current on the degree of electrolyte dissociation," Elektrokhimiya, vol. 24, no. 4, pp. 539-541, 1988.

[38] A. Sokirko and Yu. Kharkats, "the limiting di f fus ion and migration currents as functions of the rate constants of electrolyte dissociation and recombination," Elektrokhimiya, vol. 25, no. 3, pp. 331-335, 1989.

[39] D. V. Gibbs, Thermodynamics. Statistical Mechanics, Moscow, Russia, 1982.

[40] L. N. Antropov, Theoretical Electrochemistry, Moscow, Russia, 1984.

[41] H. G. Hertz, Electrochemistry, New York, NY, USA, 1980.

[42] V. Levich et al., Physicochemical Hydrodynamics, Moscow, Russia, 1959.

[43] J. Neumann, Electrochemical Systems, Moscow, Russia, 1977.

[44] V. Skorcheletti, Theoretical Electrochemistry, Leningrad, Russia, 1969.

[45] L. D. Landau and E. M. Lifshits, Theoretical Physics. Vol. 8. Electrodynamics of Continuous Media, Moscow, Russia, 1982.

[46] V. Tsurko, Tr. Inst. Mat. Nats. Akad. Nauk Belarusi, vol. 3, pp. 128-133, 1999.

[47] A. I. Shvab, Elektrichestvo, no. 4, pp. 59-67, 1994.

[48] A. I. Shvab and F. Imo, Elektrichestvo, no. 5, pp. 55-59, 1994.

[49] N. N. Grinchik et al., Vesti Nats. Akad. Nauk Belarusi, Ser. Fiz.Mat. Navuk, vol. 2, pp. 66-70, 1997.

[50] I. E. Tamm, Principles of the Theory of Electricity, Moscow, Russia, 1976.

[51] J. A. Stratton, The Theory of Electromagnetism, Leningrad, Russia, 1938.

[52] N. N. Grinchik, "Diffusional-electrical phenomena in electrolytes," Journal of Engineering Physics and Thermophysics, vol. 64, no. 5, pp. 497-504, 1993.

[53] N. N. Grinchik, "Electrodiffusion phenomena in electrolytes," Inzhenerno-Fizicheskii Zhurnal, vol. 64, no. 5, pp. 610-618, 1993.

[54] N. N. Grinchik et al., "Interaction of thermal and electric phenomena in polarized media," Mat. Modelir, vol. 12, no. 11, pp. 67-76, 2000.

[55] N. N. Grinchik, Modeling of Electrical and Thermophysical Processes in Layered Medium, Belorusskaya Nauka Press, Minsk, Russia, 2008.

[56] N. N. Grinchik, A. N. Muchynski, A. A. Khmyl, and V. A. Tsurkob, "Finite-differences method for modeling electric diffusion phenomena," Matematicheskoe Modelirovanie, vol. 10, no. 8, pp. 55-66, 1998.

[57] L. N. Antropov, Theoretical Electrochemistry, Moscow, Russia, 1989.

[58] N. A. Kostin and O. V. Labyak, Elektrokhimiya, vol. 31, no. 5, pp. 510-516, 1995.
[59] A. I. Dikusar et al., Thermokinetic Phenomena in HighFrequency Processes, Kishinev, 1989.

[60] F. Bark, Yu. Kharkats, and R. Vedin, Elektrokhimiya, vol. 34, no. 4, pp. 434-44, 1998.

[61] N. N. Grinchik and V. A. Tsurko, "Problem of modeling of the interaction of nonstationary electric, thermal, and diffusion field in layered media," Journal of Engeneering Physics and Thermodynamics, vol. 75, no. 3, pp. 693-699, 2002.

[62] P. Kolesnikov, Theory and Calculation of Waveguides, lightguides, and integral-optoelectronics elements. Electrodynamics and Theory of Waveguides, ITMO NAN Belarusi, Minsk, Russia, 2001.

[63] T. Skanavi, Dielectric Physics (Region of Weak Fields), Gostekhizdat, Moscow, Russia, 1949.

[64] P. Perre and I. W. Turner, " A complete coupled model of the combined microwave and convective drying of softwood in an oversized waveguid," in Proceedings of the 10th International Drying Symposium (IDS'96), vol. A, pp. 183-194, Krakow, Poland, 1996.

[65] J. Jaeger, Methods of Measurement in Electrochemistry, Moscow, Russia, 1977.

[66] Y. Barash and V. L. Ginzburg, "On the expressions of energy density and the release of heat in electrodynamics of a dispersing and absorbing medium," Uspekhi Fizicheskikh Nauk, vol. 118, no. 3, p. 523, 1976.

[67] D. E. Vakman and L. A. Vanshtein, "Amplitude, phase, frequency are the principal notions in the theory of ossillations," Uspekhi Fizicheskikh Nauk, vol. 123, no. 4, p. 657, 1977.

[68] B.-T. Choo, Plasma in a Magnetic Field and Direct Thermal-toElectric Energy Conversion, Moscow, Russia, 1962.

[69] I. V. Antonets, L. N. Kotov, V. G. Shavrov, and V. I. Shcheglov, "Energy characteristics of propagation of a wave through the boundaries of media with complex parameters," Radiotekhnika i Elektronika, vol. 54, no. 10, pp. 1171-1183, 2009.

[70] I. E. Tamm, Foundations of Electricity Theory, Nauka, Moscow, Russia, 2003. 

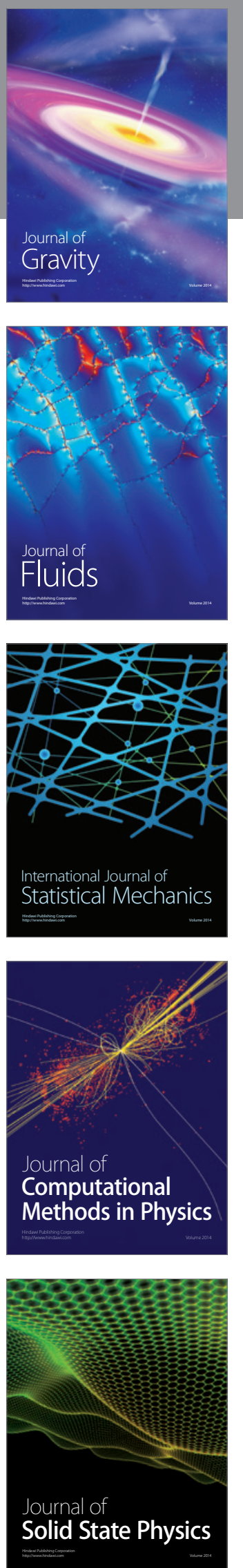

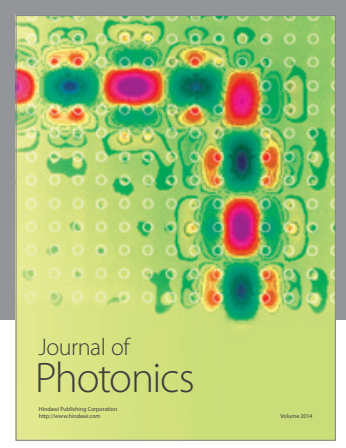

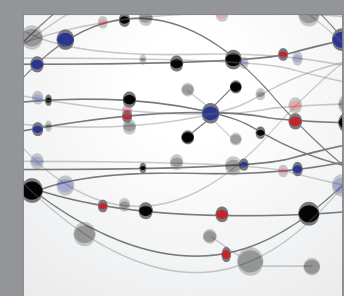

The Scientific World Journal
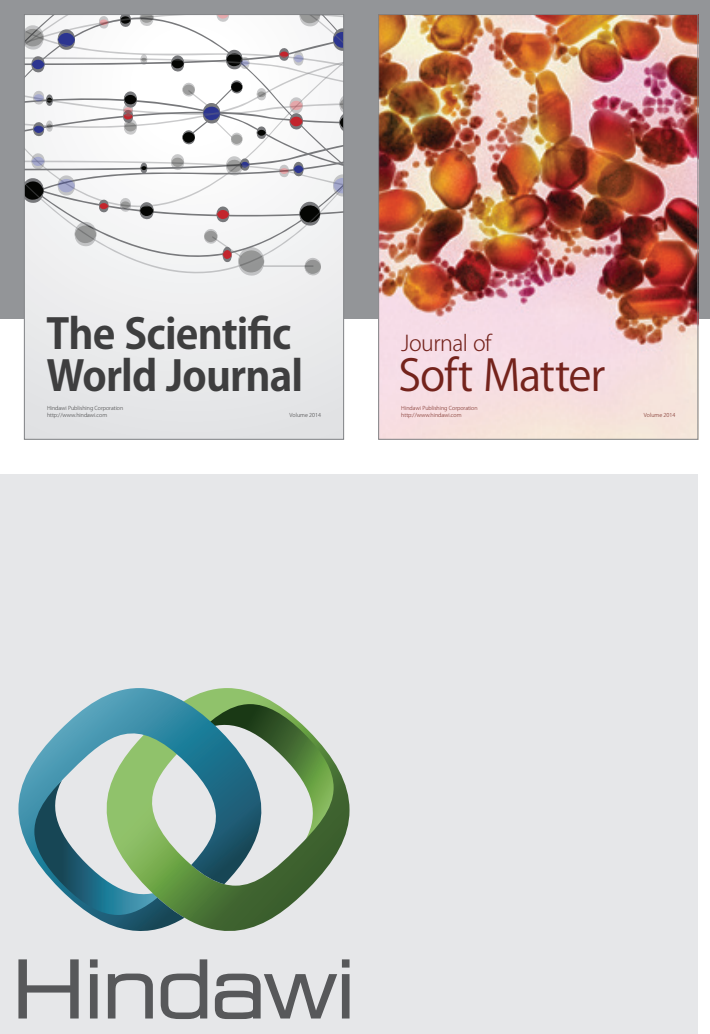

Submit your manuscripts at

http://www.hindawi.com
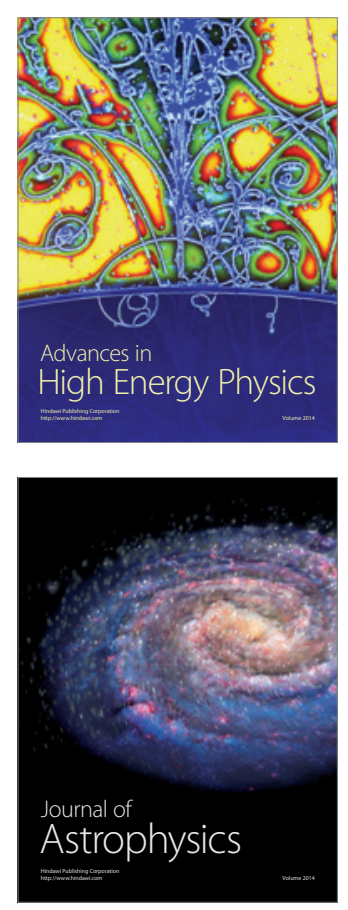
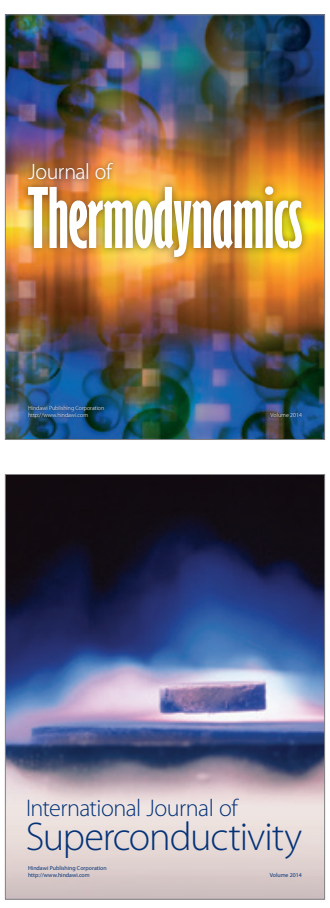
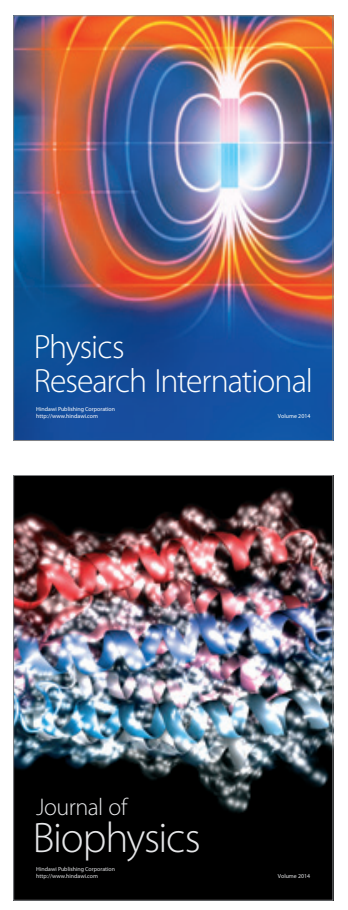
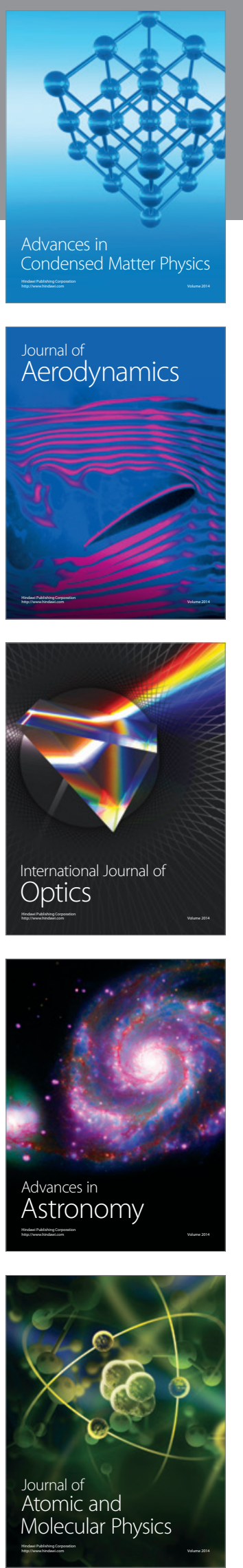